\title{
Theory of Applying Heat Flow from Thermostatted Boundary Walls: Dissipative and Local-Equilibrium Responses and Fluctuation Theorems
}

\author{
Akira Onuki \\ Department of Physics, Kyoto University, Kyoto 606-8502, Japan*
}

(Dated: August 21, 2019)

\begin{abstract}
We construct a microscopic theory of applying a heat flow from thermostatted boundary walls in the film geometry. We treat a classical one-component fluid, but our method is applicable to any fluids and solids. We express linear response of any variable $\mathcal{B}$ in terms of the time-correlation functions between $\mathcal{B}$ and the heat flows $\mathcal{J}_{K}$ from the thermostats to the particles. Furthermore, the surface variables $\mathcal{J}_{K}$ can be written in the form of space integrals of bulk quantities from the equations of motion. Owing to this surface-to-bulk relation, the steady-state response functions consist of dissipative and local-equilibrium parts, where the former gives rise to Fourier's law with Green's expression for the thermal conductivity. In the nonlinear regime, we derive the steady-state distribution in the phase space in the McLennan-Zubarev form from the first principles. Some fluctuation theorems are also presented.
\end{abstract}

\section{INTRODUCTION}

In the linear response theory in statistical mechanics, response of any physical quantity to a small perturbation is expressed in terms of appropriate time-correlation functions $\stackrel{1-4}{\underline{-4}}$. For thermal disturbances, Green ${ }^{5}$ expressed the transport coefficients such as the viscosities and the thermal conductivity in terms of the timecorrelation functions of the stress and the heat flux, respectively. These expressions also followed from the relaxations of the time-correlation functions of the hydrodynamic variables $1,2,6$. These gross variables obey the fluctuating hydrodynamic equations with random stress and heat current slightly away from equilibrium ${ }^{7}$, where the latter satisfy the fluctuation-dissipation relations -11 . Some attempts were also made to develop nonlinear theories of thermal disturbances $12-15$. On the other hand, for externally applied fields (such as magnetic field), Kubo $\underline{16}$ developed a linear response theory, where the Hamiltonian consists of the unperturbed one $\mathcal{H}$ and a small timedependent perturbation as $\mathcal{H}^{\prime}=\mathcal{H}-\gamma_{\mathrm{ex}}(t) \mathcal{A}$. Here, $\gamma_{\mathrm{ex}}(t)$ is an applied field and $\mathcal{A}$ is its conjugate variable.

Thermal disturbances are usually induced from boundaries, while the early theories $5,8,12-15,17$ started with nearly homogeneous velocity and temperature gradients in the cell interior and are not directly applicable to heterogeneous systems. Hence, we should develop a theory treating perturbations applied at boundaries. With this objective, we recently applied shear strains to particle systems by slightly moving boundary walls $\frac{18}{}$. We set up a Hamiltonian in Kubo's form, where the applied field $\gamma_{\mathrm{ex}}(t)$ is the mean shear strain in a film and the conjugate variable $\mathcal{A}$ is expressed in terms of the tangential forces from the bottom and top walls to the particles. Then, linear response of any variable $\mathcal{B}$ can be expressed in terms of the time-correlation function of $\mathcal{B}$ and $\mathcal{A}$. As

*email:onuki@scphys.kyoto-u.ac.jp a key relation, we further set $\mathcal{A}=\int d \boldsymbol{r}\left[\Pi_{x z}-z \partial J_{x} / \partial t\right]$, where $\Pi_{x z}$ is the shear stress ( $x z$ component), and $J_{x}$ is the momentum density ( $x$ component) in the bulk. This surface-to-bulk relation reveals how the surface forces induce bulk response, leading to Green's expression for the shear viscosity $\eta$. It is worth noting that Petravic and Harrowell 19 expressed $\eta$ in terms of the surface-force time-correlation.

The thermal conductivity $\lambda$ (as well as $\eta$ ) can be calculated from the Green-Kubo formula via equilibrium molecular dynamics simulation 2.3 . However, to study nonequilibrium steady states, we need at least two thermostats at different temperatures 20 . There have been a number of such nonequilibrium simulations. In particular, a heat flow was applied across solid-liquid 19,21 and gas-liquid $^{22}$ interfaces, in near-critical fluids ${ }^{23}$, and in one-dimensional lattices $24-26$. We also mention the theories on fluctuation theorems $27-35$. In their application to heat-conducting steady states $\underline{36}-\underline{41}$, relevant are the heat flows $\mathcal{J}_{K}$ from the thermostats to the particles, where $K$ represents the top or bottom thermostat in the film geometry. Then, in the linear regime, $\lambda$ can be expressed in terms of the surface variables $\mathcal{J}_{K}$. However, Green's expression for $\lambda$ is written in terms of the heat flux in the bulk ${ }^{19,20}$, so it has not been derived from the fluctuation theorems. On the other hand, the Green-Kubo formula readily follows from the fluctuation theorems for a perturbation applied in the bulk region $27,28,32,34$.

In this paper, we use Nosé-Hoover thermostats $2,3,42,43$ in top and bottom boundary layers in contact with a classical fluid. We induce heat conduction by changing the boundary temperatures by $\delta T_{K}(t)$ for $t>0$. In the linear regime, we obtain response relations of any variable $\mathcal{B}$ in terms of the time-correlation functions $\left\langle\mathcal{B}(t) \mathcal{J}_{K}(0)\right\rangle_{\mathrm{e}}$ in equilibrium. As in the case of boundary-driven strains $\frac{18}{}$, we further express $\mathcal{J}_{K}$ as a sum of bulk terms, where one term yields local-equilibrium response and another one dissipative response. Remarkably, the latter is proportional to the heat flux integral $\int d \boldsymbol{r} J_{h}^{z}(\boldsymbol{r}, t)$, giving rise to Green's expression for $\lambda$. We then calculate the relaxations of the hydrodynamic variables after a boundary 
temperature change, where we account for the adiabatic (piston) effect at a fixed cell volume $e^{44}-46$. These average deviations are related to $\left\langle\mathcal{B}(t) \mathcal{J}_{K}(0)\right\rangle_{\mathrm{e}}$. We also calculate the Evans-Searles dissipation function 27,36 analytically in transient states in the linear regime, which is related to the entropy production and the logarithm of the distribution function of the hydrodynamic variables.

In this paper, we also study nonlinear dynamics in the phase space on the basis of the research on fluctuation theorems ${ }^{27-41}$, where the boundary temperature changes $\delta T_{K}$ need not be small. It enables us to understand the linear theory itself from a wide perspective. We add some nonlinear results particularly in transient states for $\delta T_{\text {top }}=\delta T_{\text {bot }}$ and in steady states for $\delta T_{\text {top }} \neq \delta T_{\text {top }}$. In the latter finding, we obtain the steady-state distribution in the classical form of McLennan ${ }^{13}$ and Zubarev $\underline{14}$ from the first principles. Here, using the surface-to-bulk relationship, we can introduce the local-equilibrium distribution with a space-dependent temperature, from which relaxation to a steady state occurs in a microscopic time.

The organization of this paper is as follows. In Sec. II, we will present the theoretical background of our thermostatted system. In Sec.III, the linear response will be discussed using the Liouville equation. In Sec.IV, a nonlinear theory will be presented. We will also calculate the relaxation behaviors of the hydrodynamic variables in Appendix A and the dissipation function in Appendix B after a boundary temperature change. A theory of long-range correlations in the canonical ensemble $\frac{47}{}$ will be presented in Appendix C, which is crucial in calculating the local-equilibrium response.

\section{THEORETICAL BACKGROUND}

We treat a classical one-component fluid with particle number $N$ in a film cell. We write the particle positions as $\boldsymbol{r}_{i}=\left(x_{i}, y_{i}, z_{i}\right)$, the velocities as $\boldsymbol{v}_{i}=\dot{\boldsymbol{r}}_{i}=\left(v_{i}^{x}, v_{i}^{y}, v_{i}^{z}\right)$, and the momenta as $\boldsymbol{p}_{i}=m \boldsymbol{v}_{i}=\left(p_{i}^{x}, p_{i}^{y}, p_{i}^{z}\right)$ with $m$ being the particle mass. Herafter, the upper dot denotes taking the time derivative $\partial / \partial t$. The space dimensionality $d$ can also be one or two. We write the cell thickness as $H$ and the lateral dimension as $L$ in the $x y$ plane. The surface area of each boundary layer is $A=L^{d-1}$ and the cell volume is $V=H A$. We can impose the periodic boundary condition along the $x$ and $y$ axes. These lengths are much longer than the particle diameters. The fluid is in a liquid or supercritical one-phase state with a high density $n=N / V$.

\section{A. Heat variable and heat inputs}

We introduce thermostatted boundary layers 48 , which are in the regions $-\ell_{\mathrm{w}}<z<0$ at the bottom and $0<$ $z-H<\ell_{\mathrm{w}}$ at the top. To each layer, $M$ particles are bound by spring potentials $\psi\left(\left|\boldsymbol{r}_{k}-\boldsymbol{R}_{k}\right|\right)$, where $\boldsymbol{r}_{k}$ are the positions of the bound particles and $\boldsymbol{R}_{k}$ are the pinning centers fixed to the layers at a high density. We assume $\ell_{\mathrm{w}} \ll H$ and $M \ll N$ for simplicity. In our previous papers 18.48 , we used the harmonic form $\psi(r)=s_{0} r^{2} / 2$, where impenetrable walls were realized for large $s_{0}$. In one-dimensional model solids $\underline{24}-\underline{26}$, only the end particles were thermostatted (where $M=1$ ).

Particle pairs $i$ and $j$ interact via short-range potentials $\phi_{a b}\left(r_{i j}\right)$ with $r_{i j}=\left|\boldsymbol{r}_{i}-\boldsymbol{r}_{j}\right|$, where $i \in a$ and $j \in b$ with $a$ and $b$ denoting either of unbound or bound particles. We write $\phi_{i j}=\phi_{a b}\left(r_{i j}\right)$ and $\psi_{k}=\psi\left(\left|\boldsymbol{r}_{k}-\boldsymbol{R}_{k}\right|\right)$. The total energy of the particles is written as

$$
\mathcal{H}=\sum_{i} \frac{1}{2 m_{i}}\left|\boldsymbol{p}_{i}\right|^{2}+\frac{1}{2} \sum_{i, j} \phi_{i j}+\sum_{k>N} \psi_{k}
$$

where we sum over all the particles. The mass of the bound particles can differ from that of the unbound ones.

We define the number density and the energy density of the unbound particles microscopically as

$$
\begin{aligned}
& \hat{n}(\boldsymbol{r}, t)=\sum_{i \leq N} \delta\left(\boldsymbol{r}-\boldsymbol{r}_{i}\right), \\
& \hat{e}(\boldsymbol{r}, t)=\sum_{i \leq N} e_{i} \delta\left(\boldsymbol{r}-\boldsymbol{r}_{i}\right),
\end{aligned}
$$

where $e_{i}=m\left|\boldsymbol{v}_{i}\right|^{2} / 2+\sum_{j \leq N} \phi_{i j} / 2$ is the energy supported by particle $i$. Hereafter, the variables with an upper caret symbol are microscopically defined spacedependent variables. Following the literature ${ }^{2,4,6}$, we introduce the heat variable $\hat{q}(\boldsymbol{r}, t)$ by

$$
\hat{q}(\boldsymbol{r}, t)=\hat{e}(\boldsymbol{r}, t)-h \hat{n}(\boldsymbol{r}, t) .
$$

Here, $h=(e+p) / n$ is the enthalpy per particle, where $e$, $p$, and $n$ are the thermodynamic energy density, pressure, and number density, respectively. Then, the equilibrium average of $\hat{q}$ is equal to $-p$ and the entropy per particle $s$ satisfies the thermodynamic differential relation $n T d s=$ $d e-h d n$, so we can treat $\delta \hat{q}=\hat{q}+p$ as the deviation of the fluctuating entropy variable multiplied by $n T$. See the last paragraph of this subsection for more discussions on the flux of $\hat{q}$.

The unbound particles $(i \leq N)$ obey Newton's equations $m \dot{\boldsymbol{v}}_{i}=\boldsymbol{f}_{i}$ with $\boldsymbol{f}_{i}=-\partial \mathcal{H} / \partial \boldsymbol{r}_{i}$ being the force on particle $i$. Then, $\hat{q}$ evolves in time as

$$
\frac{\partial}{\partial t} \hat{q}+\nabla \cdot \boldsymbol{J}_{h}=\sum_{i \leq N} \boldsymbol{v}_{i} \cdot\left(\boldsymbol{f}_{i}^{\mathrm{top}}+\boldsymbol{f}_{i}^{\mathrm{bot}}\right) \delta\left(\boldsymbol{r}-\boldsymbol{r}_{i}\right) .
$$

where $\boldsymbol{J}_{h}(\boldsymbol{r}, t)$ is the heat flux in the cell and the right hand side represents the heat-input density from the walls. We write the forces on unbound particle $i \leq N$ from the top and bottom bound particles $(k>N)$ as

$$
\boldsymbol{f}_{i}^{\mathrm{top}}=-\sum_{k \in \mathrm{top}} \nabla_{i} \phi_{i k}, \quad \boldsymbol{f}_{i}^{\mathrm{bot}}=-\sum_{k \in \text { bot }} \nabla_{i} \phi_{i k}
$$


where $\nabla_{i}=\partial / \partial \boldsymbol{r}_{i}=\left(\nabla_{i}^{x}, \nabla_{i}^{y}, \nabla_{i}^{z}\right)$. The $\alpha$ component of $\boldsymbol{J}_{h}(\alpha=x, y, z)$ is microscopically expressed as

$$
\begin{aligned}
J_{h}^{\alpha} & =\sum_{i \leq N}\left(e_{i}-h\right) v_{i}^{\alpha} \delta\left(\boldsymbol{r}-\boldsymbol{r}_{i}\right) \\
& -\sum_{i, j \leq N} \frac{1}{2 r_{i j}} \phi_{i j}^{\prime} x_{i j}^{\alpha}\left(\boldsymbol{r}_{i j} \cdot \boldsymbol{v}_{j}\right) \hat{\delta}\left(\boldsymbol{r}, \boldsymbol{r}_{i}, \boldsymbol{r}_{j}\right),
\end{aligned}
$$

where $\phi_{i j}^{\prime}=d \phi_{i j} / d r_{i j}$ and $x_{i j}^{\alpha}$ is the $\alpha$ component of $\boldsymbol{r}_{i j}=\boldsymbol{r}_{i}-\boldsymbol{r}_{j}$. We use the Irving-Kirkwood delta function $49 \hat{\delta}\left(\boldsymbol{r}, \boldsymbol{r}_{i}, \boldsymbol{r}_{j}\right)=\int_{0}^{1} d \lambda \delta\left(\boldsymbol{r}-\lambda \boldsymbol{r}_{i}-(1-\lambda) \boldsymbol{r}_{j}\right)$, which is nonvanishing on the line segment connecting $\boldsymbol{r}_{i}$ and $\boldsymbol{r}_{j}$. The microscopic expression for the pressure is then

$$
\hat{p}=\sum_{i \leq N} \frac{\left|\boldsymbol{p}_{i}\right|^{2}}{d m} \delta\left(\boldsymbol{r}-\boldsymbol{r}_{i}\right)-\sum_{i, j \leq N} \frac{r_{i j}}{2 d} \phi_{i j}^{\prime} \hat{\delta}\left(\boldsymbol{r}, \boldsymbol{r}_{i}, \boldsymbol{r}_{j}\right)
$$

where the equilibrium average $p(T)=\langle\hat{p}\rangle_{\mathrm{e}}$ in the bulk region is the the thermodynamic pressure.

It is convenient to define the total internal energy of the unbound particles as

$$
\mathcal{H}_{\text {in }}=\sum_{i \leq N} e_{i}=\sum_{i \leq N} \frac{1}{2 m}\left|\boldsymbol{p}_{i}\right|^{2}+\frac{1}{2} \sum_{i \leq N, j \leq N} \phi_{i j} .
$$

At fixed volume $V$ and $N, \mathcal{H}_{\text {in }}$ changes in time as

$$
\dot{\mathcal{H}}_{\text {in }}=\frac{d}{d t} \int d \boldsymbol{r} \hat{q}=\mathcal{I}_{\text {top }}+\mathcal{I}_{\text {bot }}
$$

where we use $\int d \boldsymbol{r} \hat{n}=N$ and the space integral $\int d \boldsymbol{r}(\cdots)$ is taken in a region containing all the unbound particles. The $\mathcal{I}_{K}$ is the heat flow from the bound particles in the layer $K$ to the unbound particles defined as

$$
\mathcal{I}_{K}=\sum_{i \leq N} \boldsymbol{v}_{i} \cdot \boldsymbol{f}_{i}^{K}
$$

Hereafter, the subscript $K$ represents top or bottom.

Using Eq.(5) we also integrate the product $z \partial \hat{q} / \partial t$ as

$$
\begin{aligned}
\frac{d}{d t} \int d \boldsymbol{r} z \hat{q}-\int d \boldsymbol{r} J_{h}^{z} & =\sum_{K} \sum_{i \leq N} z_{i} \boldsymbol{v}_{i} \cdot \boldsymbol{f}_{i}^{\mathrm{K}} \\
& =H \mathcal{I}_{\mathrm{top}}
\end{aligned}
$$

In the first line, the left hand side is written in terms of the degrees of freedom of the unbound particles, while the right hand side involves the bound ones. In the second line, we retain the contribution at the top replacing $z_{i}$ by $H$ from $\left|z_{i}-H\right|<\ell_{\mathrm{w}} \ll H$. We thus find

$$
\mathcal{I}_{\text {top }}=\int d \boldsymbol{r} \frac{z}{H} \frac{\partial \hat{q}}{\partial t}-\frac{1}{H} \mathcal{G}, \quad \mathcal{I}_{\text {bot }}=\dot{\mathcal{H}}_{\text {in }}-\mathcal{I}_{\text {top }},
$$

where we define the integrated heat flux along the $z$ axis,

$$
\mathcal{G}(t)=\int d \boldsymbol{r} J_{h}^{z}(\boldsymbol{r}, t)
$$

In the periodic boundary condition, the surface terms are nonexistent in Eq.(12). Then, the space-time integral of the heat flux $\int_{0}^{t} d s \mathcal{G}(s)$ is equal to

$$
G_{\lambda}(t)=\sum_{i} z_{i}(t)\left[e_{i}(t)-\left\langle e_{i}\right\rangle_{\mathrm{e}}\right]
$$

This yields Helfand's formula 51,52 for the thermal conductivity $\lambda=\lim _{t \rightarrow \infty}\left[\left\langle\left[G_{\lambda}(t)-G_{\lambda}(0)\right]^{2}\right\rangle_{\mathrm{e}} / 2 t V k_{B} T^{2}\right]$, which is equivalent to Green's one in Eq. $(45)^{\frac{5}{5}}$.

Between the heat flux $J_{h}^{\alpha}(\boldsymbol{r})$ and the momentum density $J^{\alpha}(\boldsymbol{r})=\sum_{i \leq N} p_{i}^{\alpha} \delta\left(\boldsymbol{r}-\boldsymbol{r}_{i}\right)$, we can consider their equal-time correlation $G_{\alpha \beta}\left(\boldsymbol{r}-\boldsymbol{r}^{\prime}, t\right)=\left\langle J_{h}^{\alpha}(\boldsymbol{r}) J^{\beta}\left(\boldsymbol{r}^{\prime}\right)\right\rangle_{\mathrm{e}}$. It is a short-ranged function of $\boldsymbol{r}-\boldsymbol{r}^{\prime}$ and its space integral vanishes. Namely, we have $\int d \boldsymbol{r} G_{\alpha \beta}(\boldsymbol{r})=0$. This means that $J_{h}^{\alpha}(\boldsymbol{r})$ is orthogonal to the long-wavelength hydrodynamic variables, which is crucial in the projection operator formalism of irreversibility $\underline{9,10,15}$.

\section{B. Nosé-Hoover thermostats in boundary layers}

We attach Nosé-Hoover thermostats to the bound particles at the top and the bottom separately ${ }^{2,3,42,43}$. Their equations of motion are deterministic and are written as

$$
\dot{\boldsymbol{p}}_{k}=\boldsymbol{f}_{k}-\zeta_{K} \boldsymbol{p}_{k} \quad(k>N),
$$

where $\boldsymbol{p}_{k}=m_{k} \boldsymbol{v}_{k}$ and $\boldsymbol{f}_{k}=-\partial \mathcal{H} / \partial \boldsymbol{r}_{K}$ with $m_{k}$ being the mass of bound particle $k$. The coefficients $\zeta_{K}(t)$ are fluctuating friction constants obeying

$$
\tau_{\mathrm{h}}^{2} \dot{\zeta}_{K}=2 \beta E_{K} / d M-1
$$

where $\tau_{\mathrm{h}}$ is the characteristic time common to the two thermostats. In this section, we fix $\beta=1 / k_{B} T$. We assume that $\tau_{\mathrm{h}}$ is a short time independent of $T$ (see arguments around Eqs.(34) and (48)). We write the kinetic energy of the bound particles in the layer $K$ as

$$
E_{K}=\sum_{k \in K} \frac{1}{2 m_{k}}\left|\boldsymbol{p}_{k}\right|^{2}
$$

From Eq.(16) $E_{K}$ depends on $t$ as

$$
\dot{E}_{K}=\sum_{k \in K} \boldsymbol{v}_{k} \cdot \boldsymbol{f}_{k}-2 \zeta_{K} E_{K}
$$

It is convenient to introduce the total energy of the bound particles in the layer $\mathrm{K}$ as

$$
\mathcal{H}_{K}=E_{K}+\sum_{k \in K}\left[\frac{1}{2} \sum_{j \in K} \phi_{j k}+\sum_{i \leq N} \phi_{i k}+\psi_{k}\right] .
$$

The total energy of the particles in Eq.(2) is the sum,

$$
\mathcal{H}=\mathcal{H}_{\text {in }}+\mathcal{H}_{\text {top }}+\mathcal{H}_{\text {bot }}
$$

From Eqs.(11), (19), and (20) we find

$$
\dot{\mathcal{H}}_{K}=-\mathcal{I}_{K}-2 \zeta_{K} E_{K}
$$


Therefore, the heat flow from the thermostat $K$ to the particles (consisting of the bound ones in the layer $K$ and the unbound ones) is written as

$$
\mathcal{J}_{K}=\dot{\mathcal{H}}_{K}+\mathcal{I}_{K}=-2 \zeta_{K} E_{K}
$$

From Eqs.(10) and (23) $\mathcal{H}$ changes in time as

$$
\dot{\mathcal{H}}=\mathcal{J}_{\text {top }}+\mathcal{J}_{\text {bot }}
$$

From Eqs.(13), (23), and (24) we now find

$$
\begin{aligned}
& \mathcal{J}_{\text {top }}=\frac{d}{d t}\left[\int d \boldsymbol{r} \frac{z}{H} \hat{q}+\mathcal{H}_{\text {top }}\right]-\frac{1}{H} \mathcal{G}, \\
& \mathcal{J}_{\text {bot }}=\frac{d}{d t}\left[\int d \boldsymbol{r}\left(1-\frac{z}{H}\right) \hat{q}+\mathcal{H}_{\text {bot }}\right]+\frac{1}{H} \mathcal{G},
\end{aligned}
$$

which are our desired surface-to-bulk relations.

\section{Liouville equation and equilibrium distribution}

We denote all the degrees of freedom in our system by the symbol $\Gamma=\left(\boldsymbol{r}_{i}, \boldsymbol{p}_{i}, \zeta_{K}\right)$ for the $N+2 M$ particles and the two thermostats. We also introduce the sign reversal of the momenta and the friction constants,

$$
\Gamma=\left(\boldsymbol{r}_{i}, \boldsymbol{p}_{i}, \zeta_{K}\right) \rightarrow \Gamma^{*}=\left(\boldsymbol{r}_{i},-\boldsymbol{p}_{i},-\zeta_{K}\right)
$$

In our system the time-reversal symmetry holds. As a result, for each phase-space trajectory segment $\Gamma_{t}(0<$ $\left.t<t_{0}\right)$, we can conceive its time-reversed conjugate $\Gamma_{t}^{\prime}=$ $\left(\Gamma_{t_{0}-t}\right)^{*}$, where $t_{0}$ is a fixed time.

The phase-space distribution $P(\Gamma, t)$ is governed by the Liouville equation $\dot{P}+\mathcal{L} P=0$ in the Euler description in the phase space $^{3}$, where

$$
\mathcal{L}=\frac{\partial}{\partial \Gamma} \cdot \dot{\Gamma}=\sum_{i}\left[\frac{\partial}{\partial \boldsymbol{r}_{i}} \cdot \boldsymbol{v}_{i}+\frac{\partial}{\partial \boldsymbol{v}_{i}} \cdot \dot{\boldsymbol{v}}_{i}\right]+\sum_{K} \frac{\partial}{\partial \zeta_{K}} \dot{\zeta}_{K}
$$

For any initial distribution $P(\Gamma, 0)$, we obtain

$$
P(\Gamma, t)=\int d \Gamma_{0} \delta\left(\Gamma-\Gamma_{t}\right) P\left(\Gamma_{0}, 0\right),
$$

using trajectories $\Gamma_{t}$ starting from $\Gamma_{0}$ at $t=0$.

When the phase-space velocity $\dot{\Gamma}(t)$ depends only on the instantaneous position $\Gamma_{t}$ autonomously in stationary environment, $\mathcal{L}$ is independent of $t$ leading to the convenient expression $P(\Gamma, t)=\exp [-\mathcal{L} t] P(\Gamma, 0)$. Also phasespace trajectories are written as $\Gamma_{t}=\exp \left(-t \mathcal{L}^{\mathrm{T}}\right) \Gamma$ with the starting point being $\Gamma$, where $\mathcal{L}^{\mathrm{T}}=-\dot{\Gamma} \partial / \partial \Gamma$ is the transpose operator of $\mathcal{L}$. For any phase-space function $\mathcal{B}(\Gamma)$, we have $\mathcal{B}\left(\Gamma_{t}\right)=\exp \left(-t \mathcal{L}^{\mathrm{T}}\right) \mathcal{B}(\Gamma)$. Hereafter, this $\mathcal{B}\left(\Gamma_{t}\right)$ will be written simply as $\mathcal{B}(t)$. The time derivative of $\mathcal{B}$ is written as $\dot{\mathcal{B}}=-\mathcal{L}^{\mathrm{T}} \mathcal{B}$.

For stationary and homogeneous $T$, we assume that the system tends to the following equilibrium distribution,

$$
P_{\mathrm{e}}(\Gamma)=\frac{\gamma}{2 \pi} \exp \left[\beta F(T)-\beta \mathcal{H}(\Gamma)-\sum_{K} \frac{\gamma}{2} \zeta_{K}^{2}\right],
$$

where $F(T)$ is the Helmholtz free energy for all the particles. The stationality condition $\mathcal{L} P_{\mathrm{e}}=0$ holds for ${ }^{42,43}$

$$
\gamma=d M \tau_{\mathrm{h}}^{2}
$$

where the factor $d M$ appears because a single thermostat is attached to $M$ particle in each boundary layer. Hereafter, $\langle\cdots\rangle_{\mathrm{e}}=\int d \Gamma(\cdots) P_{\mathrm{e}}(\Gamma)$ denotes the equilibrium average. Then, $\left\langle\zeta_{K} \zeta_{K^{\prime}}\right\rangle_{\mathrm{e}}=\delta_{K K^{\prime}} / \gamma$ and $\left\langle\dot{\zeta}_{K} \dot{\zeta}_{K^{\prime}}\right\rangle_{\mathrm{e}}=$ $2 \delta_{K K^{\prime}} / \gamma \tau_{\mathrm{h}}^{2}$. The stationality in equilibrium gives

$$
\begin{aligned}
& \left\langle\mathcal{B}\left(t+t_{0}\right) \mathcal{C}\left(t_{0}\right)\right\rangle_{\mathrm{e}}=\langle\mathcal{B}(t) \mathcal{C}(0)\rangle_{\mathrm{e}}=\langle\mathcal{B}(0) \mathcal{C}(-t)\rangle_{\mathrm{e}} \\
& \frac{d}{d t}\langle\mathcal{B}(t) \mathcal{C}(0)\rangle_{\mathrm{e}}=\langle\dot{\mathcal{B}}(t) \mathcal{C}(0)\rangle_{\mathrm{e}}=-\langle\mathcal{B}(t) \dot{\mathcal{C}}(0)\rangle_{\mathrm{e}}
\end{aligned}
$$

Equal-time correlation functions $\langle\mathcal{B}(0) \mathcal{C}(0)\rangle_{\mathrm{e}}$ will simply be written as $\langle\mathcal{B C}\rangle_{\mathrm{e}}$, which have no time-dependence.

From Eqs. (23) and (29) we have $\left\langle\mathcal{J}_{K}\right\rangle_{\mathrm{e}}=0$ and

$$
\left\langle\mathcal{J}_{K} \mathcal{J}_{K^{\prime}}\right\rangle_{\mathrm{e}}=\delta_{K K^{\prime}}\left(k_{B} T\right)^{2}(d M+2) / \tau_{\mathrm{h}}^{2} .
$$

For $K=K^{\prime}$, the right hand side is large for large $M / \tau_{\mathrm{h}}^{2}$. In contrast, we find $\left\langle\left(\mathcal{I}_{K}\right)^{2}\right\rangle_{\mathrm{e}} \propto k_{B} \operatorname{Tn} A$ from Eq.(11), which is different from Eq.(32). If $\mathcal{B}(\{\boldsymbol{r}, \boldsymbol{p}\})$ is a variable detemined by the particle positions and momenta and not by $\zeta_{K}$, we have $\left\langle\mathcal{B} \mathcal{J}_{K}\right\rangle_{\mathrm{e}}=0$. For example, we obtain

$$
\left\langle\hat{q} \mathcal{J}_{K}\right\rangle_{\mathrm{e}}=\left\langle\mathcal{H} \mathcal{J}_{K}\right\rangle_{\mathrm{e}}=\left\langle\mathcal{H}_{K^{\prime}} \mathcal{J}_{K}\right\rangle_{\mathrm{e}}=\left\langle J_{h}^{\alpha} \mathcal{J}_{K}\right\rangle_{\mathrm{e}}=0 .
$$

However, the time-correlation functions $\left\langle\mathcal{B}(t) \mathcal{J}_{K}(0)\right\rangle_{\mathrm{e}}$ are nonvanishing for $t \gg \tau_{\mathrm{h}}$ (from Eqs.(41) and (42) below) and should be nearly equal to $\left\langle\mathcal{B}(t) \mathcal{I}_{K}(0)\right\rangle_{\mathrm{e}}$.

In the Nosé-Hoover thermostating, the time $\tau_{\mathrm{h}}$ in Eq.(17) determines the thermalization speed within the boundary layers. We assume that $\tau_{\mathrm{h}}$ is shorter than the typical microscopic time $\tau_{\mathrm{m}}$ in the bulk. Then, the temperature fluctuations of the thermostats $(\cong$ $\left.2 E_{K} / d M k_{B}-T\right)$ decay to zero quickly ${ }^{20}$. We also assume a large spring constant $\left(=d^{2} \psi / d r^{2}\right)$ to rigidly pin the bound particles on the wall18,48. Then, the potential part of $\mathcal{H}_{K}$ in Eq.(20) should also relax rapidly (see the second paragraph at the beginning of Sec.II). Under these conditions, Eqs.(19) and (23) indicate

$$
\zeta_{K} \cong-\mathcal{I}_{K} / 2 E_{K}, \quad \mathcal{J}_{K} \cong \mathcal{I}_{K}
$$

This should hold even when the boundary temperatures $T_{K}(t)$ vary in time. Thus, slow time-evolution of $\zeta_{K}(t)$ arises from that of $\mathcal{I}_{K}(t)$. Such thermostatted layers can serve as isothermal boundaries in the hydrodynamic description. In their simulation on shear flow, Hoover et al. 53 assumed the friction of the form $\zeta=\sum_{i} \boldsymbol{p}_{i} \cdot \boldsymbol{f}_{i} / \sum_{i}\left|\boldsymbol{p}_{i}\right|^{2}$ for a single thermostat coupled to all the particles as $\dot{\boldsymbol{p}}_{i}=\boldsymbol{f}_{i}-\zeta \boldsymbol{p}_{i}$. In this method, $\sum_{i}\left|\boldsymbol{p}_{i}\right|^{2}$ is exactly fixed at a constant.

\section{LINEAR RESPONSE}

Next, starting with the equilibrium distribution $P_{\mathrm{e}}(\Gamma)$ in Eq.(29) at $t=0$, we slightly change the top and bottom temperatures in Eq.(17) from $T$ to $T+\delta T_{K}(t)$ for $t>0$. We calculate linear response to $\delta T_{K}(t)$. 


\section{A. Small bondary temperature changes}

We define the mean temperature deviation $\delta T_{\mathrm{m}}$ and the mean temperature gradient $\mathcal{T}$ by

$$
\begin{aligned}
& \delta T_{\mathrm{m}}=\left(\delta T_{\text {top }}+\delta T_{\text {bot }}\right) / 2, \\
& \mathcal{T}=\left(\delta T_{\text {top }}-\delta T_{\text {bot }}\right) / H .
\end{aligned}
$$

which can depend on $t(>0)$. We fix the thermostat time $\tau_{\mathrm{h}}($ and $\gamma)$ (see discussions around Eq.(48)).

To linear order, $\mathcal{L}$ in Eq. (27) is changed by

$$
\delta \mathcal{L}(t)=\frac{2}{\gamma} \sum_{K} \delta \beta_{K}(t) E_{K} \frac{\partial}{\partial \zeta_{K}}
$$

where $\delta \beta_{K}(t) \cong-\delta T_{K}(t) / k_{B} T^{2}$. The deviation $\delta P(t)=$ $P(t)-P_{\mathrm{e}}$ obeys the linearized equation,

$$
\frac{\partial}{\partial t} \delta P(t)+\mathcal{L} \delta P(t)=-\delta \mathcal{L}(t) P_{\mathrm{e}}
$$

From $\partial P_{\mathrm{e}} / \partial \zeta_{K}=-\gamma \zeta_{K} P_{\mathrm{e}}$, we thus obtain

$$
\delta P(\Gamma, t)=-\int_{0}^{t} d s \sum_{K} \mathcal{J}_{K}(-s) \delta \beta_{K}(t-s) P_{\mathrm{e}}(\Gamma)
$$

with the aid of $e^{-s \mathcal{L}} \mathcal{J}_{K} P_{\mathrm{e}}=\mathcal{J}_{K}(-s) P_{\mathrm{e}}$ (see Eq.(31)). Here, we use backward trajectories $\Gamma_{-s}(0<s<t)$ with $\Gamma_{0}=\Gamma$ in $\mathcal{J}_{K}(-s)$. Note that the variables $\mathcal{J}_{K}$ have already appeared in Eqs.(23)-(25). From the timereversal symmetry in Eq. $(26)$, we change $\Gamma_{-s}$ to $\left(\Gamma_{s}\right)^{*}$ and $\mathcal{J}_{K}(-s)$ to $-\mathcal{J}_{K}(s)$ in Eq.(39). Then, we obtain

$$
\delta P\left(\Gamma^{*}, t\right)=\int_{0}^{t} d s \sum_{K} \mathcal{J}_{K}(s) \delta \beta_{K}(t-s) P_{\mathrm{e}}(\Gamma),
$$

using forward trajectories $\Gamma_{s}(0<s<t)$ with $\Gamma_{0}=\Gamma$.

Now, for any phase-space variable $\mathcal{B}(\Gamma)$, we consider its deviation $\delta \mathcal{B}=\mathcal{B}-\langle\mathcal{B}\rangle_{\mathrm{e}}$. From Eq.(39), its nonequilibrium average is written to linear order as ${ }^{16}$

$$
\delta \overline{\mathcal{B}}(t)=-\int_{0}^{t} d s \sum_{K} \chi_{\mathcal{B} K}(s) \delta \beta_{K}(t-s) .
$$

We use the time-correlation functions,

$$
\chi_{\mathcal{B} K}(t)=\left\langle\mathcal{B}(t) \mathcal{J}_{K}(0)\right\rangle_{\mathrm{e}}=\left\langle\mathcal{B}(0) \mathcal{J}_{K}(-t)\right\rangle_{\mathrm{e}}
$$

which should be nearly equal to $\left\langle\mathcal{B}(t) \mathcal{I}_{K}(0)\right\rangle_{\mathrm{e}}$ for $t \gg$ $\tau_{\mathrm{h}}$. For $\mathcal{B}=\mathcal{H}$, Eq. (41) gives the average energy change $\delta \overline{\mathcal{H}}(t)$, where with the aid of Eq.(24) we have

$$
\chi_{H K}(t)=\left\langle\mathcal{H}(t) \mathcal{J}_{K}(0)\right\rangle_{\mathrm{e}}=\int_{0}^{t} d s \sum_{K^{\prime}} \chi_{K K^{\prime}}(s) .
$$

For the heat flows $\mathcal{J}_{K}$, we define their time correlations,

$$
\chi_{K K^{\prime}}(t)=\left\langle\mathcal{J}_{K}(t) \mathcal{J}_{K^{\prime}}(0)\right\rangle_{\mathrm{e}}
$$

which are nearly equal to $\left\langle\mathcal{I}_{K}(t) \mathcal{I}_{K^{\prime}}(0)\right\rangle_{\mathrm{e}}$ for $t \gg \tau_{\mathrm{h}}$.

Let us consider the average heat flux $\bar{J}_{h}^{z}=-\lambda \mathcal{T}$ in the steady states with constant $\delta T_{K}$ for small boundary resistance. The coefficient $\lambda$ is the thermal conductivity,

$$
\begin{aligned}
\lambda & =\frac{H}{2 k_{B} T^{2}} \int_{0}^{\infty} d t\left\langle J_{h}^{z}(\boldsymbol{r}, t)\left[\mathcal{J}_{\mathrm{bot}}(0)-\mathcal{J}_{\mathrm{top}}(0)\right]\right\rangle_{\mathrm{e}} \\
& =\frac{1}{V k_{B} T^{2}} \int_{0}^{\infty} d t \int d \boldsymbol{r} \int d \boldsymbol{r}^{\prime}\left\langle J_{h}^{z}(\boldsymbol{r}, t) J_{h}^{z}\left(\boldsymbol{r}^{\prime}, 0\right)\right\rangle_{\mathrm{e}}
\end{aligned}
$$

The first line follows from Eq.(41), where the integrand depends on $t$ and $z$ but its time integral is independent of $z$. In the second line, we obtain Green's expression ${ }^{1-5}$ using Eq.(25) and the relation $\int_{0}^{\infty} d t\left\langle J_{h}^{z}(\boldsymbol{r}, t) \dot{\mathcal{C}}(0)\right\rangle_{\mathrm{e}}=0$ for $\mathcal{C}=\hat{q}$ and $\mathcal{H}_{K}$. The integrand in Green's expression is the flux correlation function depending on $\boldsymbol{r}-\boldsymbol{r}^{\prime}$ and $t$ in the thermodynamic limit $V \rightarrow \infty$.

When $\mathcal{B}=\hat{a}(\boldsymbol{r}, t)$ is a space-dependent variable such as $\hat{q}(\boldsymbol{r}, t)$ and $J_{h}^{z}(\boldsymbol{r}, t)$ in Eq.(41), its nonequilibrium average $\delta \bar{a}(z, t)$ and the correlation functions $\chi_{a K}(z, t)=$ $\left\langle\hat{\hat{a}}(\boldsymbol{r}, t) \mathcal{J}_{K}(0)\right\rangle_{\mathrm{e}}$ obey the hydrodynamics 6.7 and depend on $z$ and $t$ in the film geometry. Here, as will be shown in Appendix A, there are two timescales ${ }^{44}$, 46 ,

$$
t_{\mathrm{a}}=H / c, \quad t_{D}=H^{2} / 4 D
$$

where $c$ is the sound speed and $D=\lambda / C_{p}$ is the thermal diffusion constant with $C_{p}=n T(\partial s / \partial T)_{p}$ being the isobaric specific heat per unit volume. For realistic $H$ we have $t_{D} / t_{\mathrm{a}} \sim H c / D \gg 1$. Then, as a causality effect, there should be no response in the interior before arrival of sounds emitted from the boundaries. We thus predict

$$
\chi_{a K}(z, t)=0 \quad(0<c t<z \text { and } H-z) .
$$

Recently, similar causality was confirmed numerically in a low- $T$ glass for propagation of transverse sounds 18 .

We are assuming that the thermostat time $\tau_{\mathrm{h}}$ in Eq.(17) is independent of $T$. The boundary heat flows $\mathcal{J}_{K}$ in Eq.(23) then appear in the response functions in Eq.(42). This was also assumed in our simulation on glasses ${ }^{48}$ and by Lepri et al. ${ }^{24}$ in their simulation of heat conduction in one dimension. More generally, when $\tau_{\mathrm{h}}$ depends on $T, \mathcal{J}_{K}$ in Eqs.(39)-(42) is replaced by

$$
\mathcal{J}_{K}^{\prime}=\mathcal{J}_{K}-d M k_{B} T^{2}\left(\frac{\partial}{\partial T} \tau_{\mathrm{h}}^{2}\right) \zeta_{K} \dot{\zeta}_{K}
$$

where the second correction term $\left(\propto d \zeta_{K}^{2} / d t\right)$ is small for $t \gg \tau_{\mathrm{h}}$ from Eq.(34). It disappears in the time integral $\int_{0}^{\infty} d t\left\langle\mathcal{B}(t) \mathcal{J}_{K}^{\prime}(0)\right\rangle_{\mathrm{e}}$ for $\left\langle\delta \mathcal{B} \zeta_{K}^{2}\right\rangle_{\mathrm{e}}=0$.

\section{B. Dissipation function in linear regime}

We can rewrite the linear response relation (41) as

$$
\delta \overline{\mathcal{B}}(t)=\langle\mathcal{B}(t) \Omega(t)\rangle_{\mathrm{e}}
$$


where $\Omega(t)$ is given by

$$
\Omega(t)=-\int_{0}^{t} d s \sum_{K} \mathcal{J}_{K}(s) \delta \beta_{K}(s) .
$$

We here calculate this function in the linear regime with details in Appendix B. It is one example of the Evans and Searles dissipation function in their nonlinear theory 27.36 . In Sec.IVA, it will be derived in the nonlinear regime.

From Eqs.(41) and (42), we notice that the average $\bar{\Omega}(t)$ and the variance of $\Omega(t)$ are related as

$$
\bar{\Omega}(t)=\frac{1}{2}\left\langle\Omega(t)^{2}\right\rangle_{\mathrm{e}} \quad(t>0) .
$$

Since $\Omega(t)$ fluctuates, we can define its distribution,

$$
f(\omega, t)=\int d \Gamma_{0} P_{\mathrm{e}}\left(\Gamma_{0}\right) \delta(\Omega(t)-\omega) .
$$

For $\bar{\Omega}(t) \gg 1, \Omega(t)$ arises from many microscopic events, so $f(\omega, t)$ asymptotically tends to be Gaussian as

$$
f(\omega, t) \propto \exp \left[\omega / 2-\omega^{2} / 4 \bar{\Omega}(t)\right] .
$$

The magnitude of $\bar{\Omega}(t)$ increases with increasing the system size and/or the duration time $t$.

We can also obtain a bulk form of $\Omega(t)$ from Eq.(25). Using $\mathcal{G}(t)$ in Eq.(14), we write its time derivative as

$$
\begin{aligned}
\dot{\Omega}(t)= & -\int d \boldsymbol{r} \delta \bar{\beta}(z, t) \frac{\partial}{\partial t} \hat{q}(\boldsymbol{r}, t)-\sum_{K} \delta \beta_{K}(t) \dot{\mathcal{H}}_{K}(t) \\
& -\mathcal{G}(t)\left[\beta_{\text {bot }}(t)-\beta_{\mathrm{top}}(t)\right] / H .
\end{aligned}
$$

Here, we set $\delta \bar{\beta}(z, t)=\delta \beta_{\text {top }}(t) z / H+\delta \beta_{\text {bot }}(t)(1-z / H)$, which is equal to $-\delta \bar{T}(z, t) / k_{B} T^{2}$ to linear order with

$$
\delta \bar{T}(z, t)=\delta T_{\text {bot }}(t)+\mathcal{T}(t) z .
$$

The second law of thermodynamics suggests the following (not rigorously). In the first two terms in Eq.(54), $\hat{q}(\boldsymbol{r}, t)$ and $\mathcal{H}_{K}(t)$ should tend to increase locally upon heating (decrease upon cooling). Here, $\delta \bar{T}(z, t)$ represents the tempearture deviation correctly near the walls and approximately far from them even in transient states. In the third term, the integrated heat flux $\mathcal{G}(t)$ should tend to be negative (positive) when $\delta T_{\text {top }}$ is larger (smaller) than $\delta T_{\text {bot }}$. Therefore, it is very probable that $\Omega(t)$ is positive for most initial states in the canonical ensemble, especially for large systems and/or at long times.

After the typical microscopic time $t_{\mathrm{m}}$, we can calculate the nonequilibrium average $\bar{\Omega}(t)$ from the hydrodynamics (see Appendix B). Using the hydrodynamic deviation of the temperature $\delta T(\boldsymbol{r}, t)$, that of the density $\delta n(\boldsymbol{r}, t)$, and that of the velocity field $\boldsymbol{v}(\boldsymbol{r}, t)$, we can express $\bar{\Omega}(t)$ as

$$
\bar{\Omega}(t)=\int d \boldsymbol{r}\left[\frac{\rho|\boldsymbol{v}|^{2}}{2 k_{B} T}-\frac{(\Delta S)_{2}}{k_{B}}+\int_{0}^{t} d t \frac{\dot{\epsilon}_{\mathrm{th}}+\dot{\epsilon}_{\mathrm{v}}}{k_{B} T}\right]>0 .
$$

Here, $\rho|\boldsymbol{v}|^{2} / 2$ is the hydrodynamic kinetic energy density with $\rho=m n$ and $(\Delta S)_{2}$ is the negative, second-order entropy deviation written as $\frac{4,54,55}{\text {, }}$

$$
(\Delta S)_{2}=-\frac{1}{2 T^{2}} C_{V}(\delta T)^{2}-\frac{1}{2 n^{2} T \kappa_{T}}(\delta n)^{2}<0,
$$

where $C_{V}=(\partial e / \partial T)_{n}$ is the isochroic specific heat per unit volume and $\kappa_{T}=(\partial n / \partial p)_{T} / n$ is the isothermal compressibility. In the last term in Eq.(56), $\dot{\epsilon}_{\mathrm{th}}$ is the heat production rate per unit volume due to $\lambda$ and $\dot{\epsilon}_{\mathrm{v}}$ is that due to the viscosities?:

$$
\begin{gathered}
\dot{\epsilon}_{\mathrm{th}}=\lambda|\nabla \delta T|^{2} / T, \\
\dot{\epsilon}_{\mathrm{v}}=\frac{\eta}{2} \sum_{i j}\left(\nabla_{i} v_{j}+\nabla_{i} v_{i}-\frac{2}{d} \delta_{i j} \nabla \cdot \boldsymbol{v}\right)^{2}+\eta_{b}|\nabla \cdot \boldsymbol{v}|^{2},
\end{gathered}
$$

where $\nabla_{i} v_{j}=\partial v_{j} / \partial x_{i}(i, j=x, y, z), \eta$ is the shear viscosity, and $\eta_{b}$ is the bulk viscosity.

The physical meaning of $(\Delta S)_{2}$ is as follows. We treat the thermodynamic entropy $S=n s$ per unit volume as a function of the energy density $e$ and the number density $n$. We then superimpose small deviations $\delta e$ and $\delta n$ on $e$ and $n$. Up to second order we find ${ }^{4,54,55}$

$$
\begin{aligned}
(\Delta S)_{2} & =S(e+\delta e, n+\delta n)-S(e, n)-(\delta e-\mu \delta n) / T \\
& =[\delta e \delta(1 / T)-\delta n \delta(\mu / T)] / 2,
\end{aligned}
$$

where $\mu$ is the reference chemical potential and the second line leads to Eq.(57). Here, $\delta e$ and $\delta n$ are coarsegrained variables varying smoothly in space. We can also treat $\delta e, \delta n$, and $\boldsymbol{v}$ as local thermal fluctuations. For small deviations, their distribution is given by $\underline{4,54,55}$

$$
P_{\text {hyd }}=\mathcal{N} \exp \left[\int d \boldsymbol{r}\left(\frac{(\Delta S)_{2}}{k_{B}}-\frac{\rho|\boldsymbol{v}|^{2}}{2 k_{B} T}\right)\right],
$$

where $\mathcal{N}$ is the normalization factor. The tempearture fluctuation is defined by $\delta T \equiv C_{V}^{-1}\left[\delta e-(\partial e / \partial n)_{T} \delta n\right]$ with $\left\langle\delta T(\boldsymbol{r}) \delta T\left(\boldsymbol{r}^{\prime}\right)\right\rangle_{\mathrm{e}}=k_{B} T^{2} C_{V}^{-1} \delta\left(\boldsymbol{r}-\boldsymbol{r}^{\prime}\right)$. From Eqs.(56) and $(60), \bar{\Omega}(t)$ is rewritten as

$$
\bar{\Omega}(t)=-\ln \left(P_{\mathrm{hyd}} / \mathcal{N}\right)+\int_{0}^{t} d t \int d \boldsymbol{r} \beta\left(\dot{\epsilon}_{\mathrm{th}}+\dot{\epsilon}_{\mathrm{v}}\right) .
$$

In Eqs.(56), (57), and (61) time-dependence of $\delta T_{K}(t)$ can be arbitrary.

To explicitly calculate $\bar{\Omega}(t)$, we assume $\delta T_{K}(t)=$ $\theta(t) \delta T_{K}$, where $\theta(t)$ is the Heaviside step function. In the initial satage $0<t<t_{\mathrm{a}}$, the disturbances are localized near the walls and $\bar{\Omega}(t)$ grows algebraically and $\bar{\Omega}(t) \propto A$ (see Eq.(B3)). For $t>t_{\mathrm{a}}$, we calculate $\bar{\Omega}(t)$ as

$$
\frac{\bar{\Omega}(t)}{V}=\psi_{1}(\tau) C_{V} \frac{\left(\delta T_{\mathrm{m}}\right)^{2}}{k_{B} T^{2}}+\left(t+t_{D} \psi_{2}(\tau)\right) \frac{\lambda \mathcal{T}^{2}}{k_{B} T^{2}},
$$

where $\bar{\Omega}(t) \propto V=A H$. The $\psi_{1}(\tau)$ and $\psi_{2}(\tau)$ are monotonically increasing, positive functions of the scaled time $\tau=t / t_{D}=4 D t / H^{2}$ with $\psi_{1}(0)=\psi_{2}(0)=0$, 
$\psi_{1}(\infty)=1$, and $\psi_{2}(\infty)=1 / 3$. As a unique effect, $\psi_{1}(\tau)$ tends to 1 quickly on the piston time $\left(\sim t_{D} / \gamma_{\mathrm{s}}^{2}\right)$ for $\gamma_{\mathrm{s}}=C_{p} / C_{V} \gg 1$ near the criticality ${ }^{444-46}$. At long times $\left(>t_{D}\right)$, we have $\bar{\Omega}(t) \cong\left(\epsilon_{\mathrm{th}} / k_{B} T\right) V t$.

In Kubo's theory ${ }^{16}$, the disssipation function is given by $\Omega(t)=\beta \int_{0}^{t} d s \dot{\mathcal{A}}(s) \gamma_{\text {ex }}(s)=\beta(\mathcal{H}(t)-\mathcal{H}(0))$ for the total Hamiltonian $\mathcal{H}^{\prime}=\mathcal{H}-\gamma_{\text {ex }}(t) \mathcal{A}$ without thermostats, where $\gamma_{\text {ex }}(t)$ is applied for $t>0$. Then, Eq.(51) holds in the linear regime, while Eqs.(94)-(96) hold in the nonlinear regime (if the perturbation $\mathcal{H}^{\prime}-\mathcal{H}$ remains unchanged). Thus, we generally have $\bar{\Omega}(t)=\beta \delta \overline{\mathcal{H}}(t)>0$.

\section{Time-correlations with $\mathcal{J}_{K}$}

In the early hydrodynamic stage $t_{\mathrm{m}} \ll t<t_{\mathrm{a}}$, Eq.(A4) and (A5) indicate that the time-correlation functions $\chi_{H K}(t)$ and $\chi_{K K}(t)$ in Eqs.(43) and (44) behave as

$$
\chi_{H K}(t) \cong \int_{0}^{t} d s \chi_{K K}(s) \sim k_{B} T^{2} A \lambda / \sqrt{D t} .
$$

which increases as $t^{-1 / 2}$ as $t \downarrow t_{\mathrm{m}}$. Thus, $\chi_{K K}(t)$ assume large positive values for $0<t \lesssim t_{\mathrm{m}}$ as in Eq.(32), but they are negative for $t \gg t_{\mathrm{m}}$. For $K \neq K^{\prime}$, we have $\chi_{K K^{\prime}}(t)=0$ in the time range $0<t<t_{\mathrm{a}}$ for $H \gg D / c$ from Eq.(47). For $t>t_{\mathrm{a}}$, Eq.(A13) leads to

$$
\int_{0}^{t} d s \frac{\chi_{K K^{\prime}}(s)}{A k_{B} T^{2}}=\frac{\lambda \psi_{1}^{\prime}}{H \gamma_{\mathrm{s}}}+\left(2 \delta_{K K^{\prime}}-1\right)\left(1+\psi_{2}^{\prime}\right) \frac{\lambda}{H},
$$

where $\psi_{1}^{\prime}=d \psi_{1}(\tau) / d \tau$ and $\psi_{2}^{\prime}=d \psi_{2}(\tau) / d \tau$ (see Appendices A and B). The first term in Eq.(64) behaves as $\lambda\left(1-\gamma_{\mathrm{s}}^{-1}\right) / \sqrt{4 \pi D t}$ for $t_{\mathrm{a}}<t<t_{D} /\left(\gamma_{\mathrm{s}}-1\right)^{2}$ from Eq.(B7) and is continuously conected to Eq.(63).

In the limit $t \rightarrow \infty$, Eq.(64) becomes

$$
\int_{0}^{\infty} d t \chi_{K K^{\prime}}(t)=\left(2 \delta_{K K^{\prime}}-1\right) k_{B} T^{2} \lambda A / H .
$$

In terms of $\mathcal{J}_{\text {a }}=\left(\mathcal{J}_{\text {bot }}-\mathcal{J}_{\text {top }}\right) / 2$, we obtain the surface expression for $\lambda$ derived by Petravic and Harrowell ${ }^{19}$,

$$
\lambda=\frac{H}{k_{B} T^{2} A} \int_{0}^{\infty} d t\left\langle\mathcal{J}_{\mathrm{a}}(t) \mathcal{J}_{\mathrm{a}}(0)\right\rangle_{\mathrm{e}} .
$$

In Eqs.(63)-(66) we can replace $\left\langle\mathcal{J}_{K}(t) \mathcal{J}_{K^{\prime}}(0)\right\rangle_{\mathrm{e}}$ by $\left\langle\mathcal{I}_{K}(t) \mathcal{I}_{K^{\prime}}(0)\right\rangle_{\mathrm{e}}$ from Eq.(34). These integral relations stem from the hydrodynamics, while the short-time behavior of $\chi_{K K}(t)$ depends on the thermostating method. In Eqs.(65) and (66) (and the first line of Eq.(45)), the main contributions arise from the long time range $t \sim t_{D}$.

To explain the thermal resistance of a solid-liquid interface in ${ }^{3} \mathrm{He}$, Puech et al. ${ }^{50}$ expressed the Kapitza length $\ell_{\mathrm{K}}$ as $\lambda / \ell_{\mathrm{K}}=\int_{0}^{\infty} d t\langle\mathcal{J}(t) \mathcal{J}(0)\rangle_{\mathrm{e}} /\left(k_{B} T^{2} A\right)$ using the Onsager theory ${ }^{8,55}$, where $\mathcal{J}(t)$ is the microscopic heat flow through the interface. Barrat and Chiaruttini21 calculated the integral $G(t)=\int_{0}^{t} d s\langle\mathcal{J}(s) \mathcal{J}(0)\rangle_{\mathrm{e}} / k_{B} T^{2} A$ at a surface between a solid and a Lennard-Jones liquid, which assumed a plateau after a microscopic time and decayed slowly. They identified the plateau as $\lambda / \ell_{\mathrm{K}}$, while Eq.(65) indicates $\lim _{t \rightarrow \infty} G(t)=\lambda / H$ for finite $H$. This aspect should further be investigated in future.

\section{Steady-state relations using surface heat flows}

In the steady state at constants $\delta T_{K}$, the expression for the average follows from Eqs.(23) and (41) as

$$
\delta \overline{\mathcal{B}}=-\sum_{K}\left\langle\delta B\left(U_{K \infty}+\delta \mathcal{H}_{K}\right)\right\rangle_{\mathrm{e}} \delta \beta_{K} .
$$

Using backward trajectories $\Gamma_{-s}$ with $\Gamma_{0}=\Gamma$, we define

$$
U_{K \infty}=\int_{0}^{\infty} d s e^{-\epsilon s} \mathcal{I}_{K}(-s),
$$

where $\epsilon$ is a positive small number ensuring convergence of the time integral. Up to linear order, the steady-state distribution $P_{\text {st }}(\Gamma)$ is expressed as

$$
P_{\mathrm{st}}(\Gamma) / P_{\mathrm{e}}(\Gamma)=1-\sum_{K}\left(U_{K \infty}+\delta \mathcal{H}_{K}\right) \delta \beta_{K} .
$$

In particular, if $\delta \beta_{\text {top }}=\delta \beta_{\text {bot }}=-\delta T / k_{B} T^{2}, P_{\text {st }}(\Gamma)$ is the new equlibrium distribution with the shifted temperature $T+\delta T$, since Eq.(10) gives $\sum_{K} U_{K \infty}=\mathcal{H}_{\text {in }}-\left\langle\mathcal{H}_{\text {in }}\right\rangle_{\mathrm{e}}$. From the time reveral symmetry, we also obtain

$$
P_{\mathrm{st}}\left(\Gamma^{*}\right) / P_{\mathrm{e}}(\Gamma)=1-\sum_{K}\left(U_{K \infty}^{\prime}+\delta \mathcal{H}_{K}\right) \delta \beta_{K},
$$

where forward trajectories appear as in Eq.(40) with

$$
U_{K \infty}^{\prime}=-\int_{0}^{\infty} d s e^{-\epsilon s} \mathcal{I}_{K}(s)
$$

We also consider the steady-state averages $\overline{\mathcal{J}}_{K}, \overline{\mathcal{I}}_{K}$, and $\bar{J}_{h}^{z}$. From Eq.(5) we find

$$
\frac{d}{d z} \bar{J}_{h}^{z}(z)=\left\langle\sum_{i \leq N} \boldsymbol{v}_{i} \cdot\left(\boldsymbol{f}_{i}^{\mathrm{top}}+\boldsymbol{f}_{i}^{\mathrm{bot}}\right) \delta\left(z-z_{i}\right)\right\rangle_{\mathrm{s}} \frac{1}{A},
$$

where we set $\delta\left(\boldsymbol{r}-\boldsymbol{r}_{i}\right) \rightarrow \delta\left(z-z_{i}\right) / A$ and $\langle\cdots\rangle_{\mathrm{s}}$ denotes the steady-state average. Thus, $\bar{J}_{h}^{z}$ is a constant in the interior but depends on $z$ near the boundaries decaying to zero in the walls. Integrating Eq.(72) across the boundaries and using Eqs.(13) and (25), we obtain

$$
\overline{\mathcal{J}}_{\text {top }}=\overline{\mathcal{I}}_{\text {top }}=-A \bar{J}_{h}^{z}, \quad \overline{\mathcal{J}}_{\text {bot }}=\overline{\mathcal{I}}_{\text {bot }}=A \bar{J}_{h}^{z} .
$$

where $\bar{J}_{h}^{z}=-\lambda \mathcal{T}$ for negligible boundary resistance.

\section{E. Steady-state bulk relations}

Using Eq.(25), we obtain the bulk expression for the steady-state average,

$$
\delta \overline{\mathcal{B}}=\int d \boldsymbol{r}^{\prime} \gamma_{B}\left(\boldsymbol{r}^{\prime}\right) \delta \bar{T}\left(z^{\prime}\right)+\sum_{K} \gamma_{B K} \delta T_{K}-\chi_{B}^{h} \mathcal{T}
$$


where $\delta \bar{T}(z)$ is the temperature profile in Eq.(54). The first two terms are local-equilibrium parts written in terms of equal-time correlations as

$$
\begin{aligned}
& \gamma_{B}(\boldsymbol{r})=\langle\delta \mathcal{B} \delta \hat{q}(\boldsymbol{r})\rangle_{\mathrm{e}} / k_{B} T^{2} \\
& \gamma_{B K}=\left\langle\delta \mathcal{B} \delta \mathcal{H}_{K}\right\rangle_{\mathrm{e}} / k_{B} T^{2}
\end{aligned}
$$

which vanish if $\mathcal{B}$ is odd with respect to the time reversal.

The third term in Eq.(74) is dissipative. In terms of $\mathcal{G}(t)$ in Eq.(14), $\chi_{B}^{h}$ is given by

$$
\chi_{B}^{h}=\int_{0}^{\infty} d t\langle\delta \mathcal{B}(t) \mathcal{G}(0)\rangle_{\mathrm{e}} / k_{B} T^{2}
$$

which yields Green's expression (45) for $\mathcal{B}=\mathcal{G}$. If $\mathcal{B}(t)$ is a long-wavelength hydrodynamic variable, it evolves slowly in time remaining nearly orthogonal to $J_{h}^{x}(0)$. For this case, we can neglect the dissipative term in Eq.(74) at long wavelengths 9.10 .

We further examine the first term in Eq.(74) when $\mathcal{B}=$ $\hat{a}(\boldsymbol{r})$ is a space-dependent variable having the even timereversal symmetry. In this case, we should replace $\gamma_{B}\left(\boldsymbol{r}^{\prime}\right)$ by the two-point equal-time correlation function,

$$
\gamma_{a}\left(\boldsymbol{r}, \boldsymbol{r}^{\prime}\right)=\left\langle\delta \hat{a}(\boldsymbol{r}) \delta \hat{q}\left(\boldsymbol{r}^{\prime}\right)\right\rangle_{\mathrm{e}} / k_{B} T^{2},
$$

while the second term $\left(\propto\left\langle\delta \hat{a}(\boldsymbol{r}) \delta \mathcal{H}_{K}\right\rangle_{\mathrm{e}}\right)$ in Eq.(74) vanishes far from the boundaries. We treat the equilibrium average $a \equiv\langle\hat{a}\rangle_{\mathrm{e}}$ as a thermodynamic quantity depending $T$ and $p$. In Appendix $\mathrm{C}$, we will find the behavior,

$$
\gamma_{a}\left(\boldsymbol{r}, \boldsymbol{r}^{\prime}\right)=g_{a}\left(\boldsymbol{r}-\boldsymbol{r}^{\prime}\right)+\left(\frac{\partial a}{\partial p}\right)_{T}\left(\frac{\partial p}{\partial T}\right)_{n} \frac{1}{V}
$$

where $\boldsymbol{r}$ and $\boldsymbol{r}^{\prime}$ are far from the boundaries. The function $g_{a}(\boldsymbol{r})$ is short-ranged satisfying

$$
\int d \boldsymbol{r} g_{a}(\boldsymbol{r})=\left(\frac{\partial a}{\partial T}\right)_{p}
$$

The second term in Eq.(79) is proportional to $V^{-1}$. Thus,

$$
\int d \boldsymbol{r} \gamma_{a}\left(\boldsymbol{r}, \boldsymbol{r}^{\prime}\right)=\left(\frac{\partial a}{\partial T}\right)_{n}
$$

We assume that the correlation length of $g_{a}(\boldsymbol{r})$ is much shorter than $H$. The long-range behavior $\left(\propto V^{-1}\right)$ in the density correlation functions has been studied in the canonical ensemble 47,56-58. In Appendix C, we will examine it for general correlation functions $\left\langle\delta \hat{a}(\boldsymbol{r}) \delta \hat{b}\left(\boldsymbol{r}^{\prime}\right)\right\rangle_{\mathrm{e}}$.

We now substitute Eq.(79) into the first term in Eq.(74). Using Eq.(80) we obtain the local-equilibrium part of the steady-state average far from the boundaries,

$$
\delta \bar{a}_{\mathrm{loc}}(z)=\left(\frac{\partial a}{\partial T}\right)_{p} \delta \bar{T}(z)+\left(\frac{\partial a}{\partial p}\right)_{T} \delta \bar{p},
$$

where $\delta \bar{T}\left(z^{\prime}\right)$ is replaced by $\delta \bar{T}(z)$ in $\int d \boldsymbol{r}^{\prime} g_{a}\left(\boldsymbol{r}-\boldsymbol{r}^{\prime}\right) \delta \bar{T}\left(z^{\prime}\right)$ and $\delta \bar{p}$ is the homogeneous pressure deviation,

$$
\delta \bar{p}=(\partial p / \partial T)_{n} \delta T_{\mathrm{m}}
$$

If $\hat{a}$ is equal to $\hat{p}$ in Eq.(8), its average deviation is equal to the above $\delta \bar{p}$. For $\hat{a}=\hat{n}$ and $\hat{q}$, we obtain

$$
\begin{aligned}
& \delta \bar{n}(z)=(\partial n / \partial T)_{p}\left(\delta \bar{T}(z)-\delta T_{\mathrm{m}}\right) \\
& n T \delta \bar{s}(z)=C_{p}\left(\delta \bar{T}(z)-\delta T_{\mathrm{m}}\right)+C_{V} \delta T_{\mathrm{m}}
\end{aligned}
$$

Thus, the space average of $\delta \bar{n}$ is zero and that of $n T \delta \bar{s}$ is $C_{V} \delta T_{\mathrm{m}}$. We confirm that $\delta \bar{T}(z)$ introduced in Eq.(55) is the local temperature deviation in steady states.

As another application of Eq.(77), we can set $\mathcal{B}(t)=$ $\hat{a}(\boldsymbol{r}, t) \hat{b}\left(\boldsymbol{r}^{\prime}, t\right)$, where $\hat{a}$ and $\hat{b}$ are hydrodynamic variables. Then, we obtain the steady-state pair correlation $g_{a b}^{\mathrm{s}}\left(\boldsymbol{r}, \boldsymbol{r}^{\prime}\right)=\left\langle\hat{a}(\boldsymbol{r}, t) \hat{b}\left(\boldsymbol{r}^{\prime}, t\right)\right\rangle_{\mathrm{s}}$. To linear order in $\mathcal{T}$, it depends only on $r=\left|\boldsymbol{r}-\boldsymbol{r}^{\prime}\right|$ far from the boundaries and its deviation is long-ranged as $\mathcal{T} r^{-1}$ for $d=3$ and as $\mathcal{T} \ln r$ for $d=2$ due to the mode-coupling effect $4,15,17,59,60$.

\section{NONLINEAR THEORY}

We finally study nonlinear dynamics, where $\delta T_{K}(t)$ are time-dependent and need not small.

\section{A. Phase-space distribution}

The trajectory equation is non-autonomaous as

$$
\dot{\Gamma}_{t}=\mathcal{V}\left(\Gamma_{t}, t\right)
$$

where $\mathcal{V}(\Gamma, t)$ is the phase-space velocity in the Euler description. It depends on $t$ in addition to $\Gamma$, since the equations for $\zeta_{K}(t)$ are changed from Eq.(17) to

$$
\tau_{\mathrm{h}}^{2} \dot{\zeta}_{K}(t)=2 \beta_{K}(t) E_{K}(t) / d M-1,
$$

where $\beta_{K}(t)=1 / k_{B}\left[T+\delta T_{K}(t)\right]$ and $\tau_{\mathrm{h}}$ is a constant.

The phase-space distribution $P(\Gamma, t)$ obeys the Liouville equation and is still expressed in the form of Eq.(28) in terms of the initial distribution $P(\Gamma, 0)$. Using trajectories starting from $\Gamma_{0}$ and reaching $\Gamma_{t}$, we find

$$
P\left(\Gamma_{t}, t\right)=e^{-\Xi(t)} P\left(\Gamma_{0}, 0\right) .
$$

Here, $e^{\Xi(t)}$ is equal to the Jacobian $d \Gamma_{t} / d \Gamma_{0}$, where the phase-space volume element moves from $d \Gamma_{0}$ to $d \Gamma_{t}$. We can then rewrite Eq.(88) as

$$
d \Gamma_{t} P\left(\Gamma_{t}, t\right)=d \Gamma_{0} P\left(\Gamma_{0}, 0\right) .
$$

Generally, $\dot{\Xi}(t)$ is equal to the time integral of the phasespace expansion factor $\Lambda(t)$ as

$$
\Xi(t)=\int_{0}^{t} d s \Lambda(s) .
$$

where $\Lambda(t)=\Lambda\left(\Gamma_{t}, t\right)$ with $\Lambda(\Gamma, t)=(\partial \mathcal{V} / \partial \Gamma)_{t}$. For the Nosé-Hoover thermostating, Eq.(16) gives

$$
\Lambda(t)=-d M \sum_{K} \zeta_{K}(t)
$$


For any initial distribution $P(\Gamma, 0)$, Evans and Searles defined the dissipation function $\Omega(t)$ by 27,36

$$
\ln \left[P\left(\Gamma_{0}, 0\right) / P\left(\Gamma_{t}, 0\right)\right]=\Xi(t)+\Omega(t) .
$$

Let $P(\Gamma, 0)$ be equal to the equilibrium distribution $P_{\mathrm{e}}(\Gamma)$ in Eq.(29). Then, time derivative of Eq.(92) gives

$$
\begin{aligned}
\dot{\Omega}(t) & =\frac{d}{d t}\left[\beta \mathcal{H}(t)+\sum_{K} \frac{\gamma}{2} \zeta_{K}(t)^{2}\right]-\Lambda(t) \\
& =-\sum_{K} \delta \beta_{K}(t) \mathcal{J}_{K}(t),
\end{aligned}
$$

where $\mathcal{H}(t)=\mathcal{H}\left(\Gamma_{t}\right)$. This agrees with Eq.(50). We find

$$
d \Gamma_{t} P_{\mathrm{e}}\left(\Gamma_{t}\right)=d \Gamma_{0} P_{\mathrm{e}}\left(\Gamma_{0}\right) \exp [-\Omega(t)] .
$$

Phase-space integration of Eq.(94) gives

$$
\left\langle e^{-\Omega(t)}\right\rangle_{\mathrm{e}}=\int d \Gamma_{0} P_{\mathrm{e}}\left(\Gamma_{0}\right) e^{-\Omega(t)}=1 .
$$

Here, if $e^{-\Omega(t)}$ is expanded with respect to $\Omega(t)$, we obtain $\langle\Omega(t)\rangle_{\mathrm{e}}=\left\langle\Omega(t)^{2}\right\rangle_{\mathrm{e}} / 2+\cdots$, which coincides with Eq.(51) in second order. We also find $\bar{\Omega}(t)>0$ since $e^{-x}+x \geq 1$ holds for any $x$. From Eqs.(89) and (94) we also obtain

$$
P(\Gamma, t)=P_{\mathrm{e}}(\Gamma) \exp [\Omega(t)],
$$

where the trajectories $\Gamma_{s}(0<s<t)$ in $\Omega(t)$ end at $\Gamma_{t}=\Gamma$ with the initial points $\Gamma_{0}$ being a function of $\Gamma$. Note that $\Omega(t)$ satisfies Eqs.(94)-(96) for any $P(\Gamma, 0)$. See another choice of $P(\Gamma, 0)$ in Sec.IVE.

Evans and Searles $27,36,38$ obtained a different form of $\Omega(t)$, where $\mathcal{J}_{K}(t)=-2 E_{K}(t) \zeta_{K}(t)$ in our $\Omega(t)$ is replaced by $-d M k_{B} T_{K}(t) \zeta_{K}(t)$. Thus, there is no essential difference between our $\Omega(t)$ and theirs on long timescales $\left(\gg \tau_{h}\right)$ from Eq.(34). In our scheme, their result exactly follows for $\tau_{\mathrm{h}}^{2} \propto T^{-1}$. In Appendix $\mathrm{D}$, we will derive $\Omega(t)$ generally including $T$-dependence of $\tau_{\mathrm{h}}$.

\section{B. Transition between equilibrium states}

Let the two boundary walls have the same temperature $T(t)=T+\delta T(t)$ with $\delta T(0)=0$, which tends to the final one $T_{\mathrm{f}}=T+\delta T_{\mathrm{f}}$ for $t \gg t_{\mathrm{ex}}$. We set $\delta \beta(t) \equiv \delta \beta_{\mathrm{top}}(t)=$ $\delta \beta_{\text {bot }}(t)$ and use Eq.(24) to find the simple form,

$$
\Omega(t)=-\int_{0}^{t} d s \dot{\mathcal{H}}(s) \delta \beta(s) .
$$

It is convenient to define the following excess function,

$$
\Omega_{\mathrm{ex}}(t)=\Omega(t)+\mathcal{H}(t) \delta \beta(t)=\int_{0}^{t} d s \mathcal{H}(s) \dot{\beta}(s),
$$

where $\dot{\beta}(t)=d \beta(t) / d t$ and $\Omega_{\mathrm{ex}}=\mathcal{H}(0) \delta \beta$ for the stepwise change. If $\dot{\beta}(t)=0$ for $t>t_{\mathrm{ex}}, \Omega_{\mathrm{ex}}(t)$ is independent of $t$ for $t>t_{\text {ex }}$ for each $\Gamma_{0}$. We rewrite Eq.(94) as

$$
d \Gamma_{t} P_{\mathrm{e}}\left(\Gamma_{t}\right) e^{-\mathcal{H}(t) \delta \beta(t)}=d \Gamma_{0} P_{\mathrm{e}}\left(\Gamma_{0}\right) e^{-\Omega_{\mathrm{ex}}(t)} .
$$

The left hand side is proportional to the canonical distribution at the temperature $T(t)$, which is expressed as

$$
P_{\mathrm{e}}(\Gamma ; T(t))=P_{\mathrm{e}}(\Gamma) \exp [\mathcal{F}(t)-\mathcal{H}(\Gamma) \delta \beta(t)] .
$$

Here, the factor $\exp [\mathcal{F}(t)]$ arises from the normalization condition. Phase-space integration of Eq.(99) gives

$$
\begin{aligned}
\mathcal{F}(t) & =\beta(t) F(T(t))-\beta F(T) \\
& =-\ln \left[\left\langle e^{-\Omega_{\mathrm{ex}}(t)}\right\rangle_{\mathrm{e}}\right],
\end{aligned}
$$

where $F(T)$ is the Helmholtz free energy for all the particles. More generally, for any variable $\mathcal{B}(\Gamma)$, we consider its equilibrium average at the temperature $T(t)$, written as $b(T(t))=\int d \Gamma \mathcal{B}(\Gamma) P_{\mathrm{e}}(\Gamma ; T(t))$, which changes from $b(T)$ at the initial temperature $T$ to $b\left(T_{\mathrm{f}}\right)$ at the final one $T_{\mathrm{f}}$ as $t$ increases. We multiply Eq.(99) by $\mathcal{B}(t)=\mathcal{B}\left(\Gamma_{t}\right)$ and perform its phase-space integration to obtain

$$
\begin{aligned}
b(T(t)) & =e^{\mathcal{F}(t)}\left\langle\mathcal{B}(t) e^{-\Omega_{\mathrm{ex}}(t)}\right\rangle_{\mathrm{e}} \\
& =b(T)+e^{\mathcal{F}(t)}\left\langle\delta \mathcal{B}(t) e^{-\Omega_{\mathrm{ex}}(t)}\right\rangle_{\mathrm{e}}
\end{aligned}
$$

where $\delta \mathcal{B}(t)=\mathcal{B}(t)-b(T)$. In Eqs.(101) and (102), the average is over $P_{\mathrm{e}}\left(\Gamma_{0}\right)$ with trajctories starting from $\Gamma_{0}$. We can confirm Eq.(102) to linear order using $\langle\delta \mathcal{B}(t)\rangle_{\mathrm{e}} \cong$ $\langle\delta \mathcal{B}(t) \Omega(t)\rangle_{\mathrm{e}}=\left\langle\delta \mathcal{B}(t) \Omega_{\mathrm{ex}}(t)\right\rangle_{\mathrm{e}}-\langle\delta \mathcal{B H}\rangle_{\mathrm{e}} \delta \beta(t),\langle\delta \mathcal{B H}\rangle_{\mathrm{e}}=$ $k_{B} T^{2}(\partial b / \partial T)_{n}$, and $e^{-\Omega_{\mathrm{ex}}} \cong 1-\Omega_{\mathrm{ex}}$.

Previously, Williams et al ${ }^{38}$ obtained some general relations and one of them is equivalent to Eq.(101). We note that Eq.(101) resembles Jarzynski's equality ${ }^{29}$ for isothermal transitions between two equilibrium states.

\section{Time reversal}

For each trajectory segment $\Gamma_{s}(0<s<t)$, we can concieve its time-reversed conjugate: $\Gamma_{s}^{\prime}$ with $\Gamma_{0}^{\prime}=\left(\Gamma_{t}\right)^{*}$ and $\Gamma_{t}^{\prime}=\left(\Gamma_{0}\right)^{*}$ at fixed $t$ (see Eq. (26)). In the present non-stationary (non-autonomous) situation, the timereversed friction variables, written as $\zeta_{K}^{\prime}(s)$, obey

$$
\tau_{\mathrm{h}}^{2} \frac{d}{d s} \zeta_{K}^{\prime}(s)=2 \beta_{K}(t-s) E_{K}^{\prime}(s) / d M-1 .
$$

Here, $\beta_{K}(t-s)$ appear. Then, the time-reversed dissipation function $\Omega^{\prime}(t)$ is just equal to $-\Omega(t)$, where $\Omega(t)$ is the original one. Assuming the equilibrium distribution $P_{\mathrm{e}}\left(\Gamma_{0}^{\prime}\right)\left(=P_{\mathrm{e}}\left(\Gamma_{t}\right)\right)$ for the initial points $\Gamma_{0}^{\prime}$, we define

$$
f_{r}(\omega, t)=\int d \Gamma_{0}^{\prime} P_{\mathrm{e}}\left(\Gamma_{0}^{\prime}\right) \delta\left(\Omega^{\prime}(t)-\omega\right) .
$$

Here, $d \Gamma_{0}^{\prime} P_{\mathrm{e}}\left(\Gamma_{0}^{\prime}\right)=d \Gamma_{t} P_{\mathrm{e}}\left(\Gamma_{t}\right)=d \Gamma_{0} P_{\mathrm{e}}\left(\Gamma_{0}\right) e^{-\Omega(t)}$ from Eq. (94) and $\delta\left(\Omega^{\prime}(t)-\omega\right)=\delta(\Omega(t)+\omega)$ from $\Omega^{\prime}(t)=$ $-\Omega(t)$. Then, $f_{r}(\omega, t)$ is related to $f(\omega, t)$ in Eq. $(52)$ as

$$
f_{r}(\omega, t)=e^{\omega} f(-\omega, t),
$$

which is a well-known result $\underline{30,31,39}$. 
Furthermore, we find $f_{r}(\omega, t)=f(\omega, t)$ when

$$
\delta \beta_{K}(s)=\delta \beta_{K}(t-s) \quad(0<s<t) .
$$

This holds for stepwise changes. Under Eq.(106), we have

$$
f(\omega, t)=e^{\omega} f(-\omega, t) .
$$

This is the transient fluctuation theorem by Evans and Searles $27,34,36$, which is exact for stepwise changes of external parameters. It was checked in simulations of small systems $27,36,38$. We can also realize Eq.(106) for periodic $\delta T_{K}(t)$, where Eq.(107) holds for particular $t$. In the nonlinear regime, $f(\omega, t)$ can significantly deviate from the Gaussian form in Eq.(53) at small $t$ due to events with large $|\Omega(t)| 33,35,41$. When the Gaussian form is nearly realized with $\bar{\Omega}(t) \gg 1$, we have $e^{-\omega} f(\omega, t) \propto$ $\exp \left[-(\omega+\bar{\Omega})^{2} / 4 \bar{\Omega}\right]$, so the equality (95) holds due to rare events with largely negative $\Omega(t)(\cong-\bar{\Omega}(t))$.

Furthermore, under Eq.(106), Eqs.(28) and (95) give

$$
P\left(\Gamma^{*}, t\right)=P_{\mathrm{e}}(\Gamma) e^{-\Omega(t)},
$$

where we use forward trajectories $\Gamma_{s}^{\prime}$ starting from $\Gamma_{0}^{\prime}=$ $\Gamma$ in $\Omega(t)$. For example, from Eq.(108), the average of the heat flow $J_{h}^{z}(\boldsymbol{r}, t)$ in Eq. (7) is written as

$$
\bar{J}_{h}^{z}(z, t)=-\left\langle J_{h}^{z}(\boldsymbol{r}, 0) e^{-\Omega(t)}\right\rangle_{\mathrm{e}}
$$

which obeys the hydrodynamics and satisfies Eq.(47).

\section{Local-equilibrium distribution}

For the stepwise boundary-temperature change, we have obtained Eqs.(107)-(109). We can further perform time-integration of Eq.(54). Some calculations give

$$
\Omega(t)=\Psi\left(\Gamma_{0}\right)-\Psi\left(\Gamma_{t}\right)+\mathcal{D}(t) .
$$

Using $\mathcal{G}(t)$ in Eq.(14) we introduce

$$
\begin{gathered}
\Psi(\Gamma)=\int d \boldsymbol{r} \delta \bar{\beta}(z) \hat{q}(\boldsymbol{r} ; \Gamma)+\sum_{K} \delta \beta_{K} \mathcal{H}_{K}(\Gamma), \\
\mathcal{D}(t)=-\gamma_{\mathrm{a}} \int_{0}^{t} d s \mathcal{G}(s)
\end{gathered}
$$

Here, we write $\hat{q}\left(\boldsymbol{r} ; \Gamma_{t}\right)=\hat{q}(\boldsymbol{r}, t)$ to avoid confusion and the coefficients $\delta \bar{\beta}(z)$ and $\gamma_{\mathrm{a}}$ are defined by

$$
\delta \bar{\beta}(z)=\delta \beta_{\mathrm{bot}}-\gamma_{\mathrm{a}} z, \quad \gamma_{\mathrm{a}}=\left(\beta_{\mathrm{bot}}-\beta_{\mathrm{top}}\right) / H,
$$

where $\delta \bar{\beta}(z) \cong-\delta \bar{T}(z) / k_{B} T^{2}$ and $\gamma_{\mathrm{a}} \cong \mathcal{T} / k_{B} T^{2}$ in linear order with $\delta \bar{T}(z)$ being given by Eq.(55). The $\mathcal{D}(t)$ represents the entropy production in the bulk region.

Using $\Psi(\Gamma)$ in Eq.(111) we can define the localequilibrium distribution at fixed $N$ and $V$ in the form,

$$
P_{\mathrm{lc}}(\Gamma)=C_{\mathrm{lc}} P_{\mathrm{e}}(\Gamma) \exp [-\Psi(\Gamma)]
$$

where the local inverse temperature is given by $\beta+\delta \bar{\beta}(z)$ and $C_{\mathrm{lc}}$ is the normalization constant determined by

$$
C_{\mathrm{lc}}=\left\langle e^{-\Psi(\Gamma)}\right\rangle_{\mathrm{e}}^{-1}=\left\langle e^{\Psi(\Gamma)}\right\rangle_{\mathrm{lc}}
$$

Hereafter, $\langle\cdots\rangle_{\mathrm{lc}}$ denotes the average over $P_{\mathrm{lc}}(\Gamma)$. Then, using Eqs.(111) and (114), we rewrite Eq.(94) as

$$
d \Gamma_{t} P_{\mathrm{lc}}\left(\Gamma_{t}\right)=d \Gamma_{0} P_{\mathrm{lc}}\left(\Gamma_{0}\right) \exp [-\mathcal{D}(t)],
$$

As in Eq.(95), phase-space integration of Eq.(116) yields

$$
\left\langle e^{-\mathcal{D}(t)}\right\rangle_{\mathrm{lc}}=\int d \Gamma_{0} P_{\mathrm{lc}}\left(\Gamma_{0}\right) e^{-\mathcal{D}(t)}=1,
$$

which yields $\overline{\mathcal{D}}(t)=\int d \omega \omega f_{\mathrm{lc}}(\omega, t)>0$, supporting the second law of thermodynamics in the bulk region.

\section{E. Steady-state distribution in nonlinear regime}

To seek the steady-state distribution $P_{\text {st }}(\Gamma)$, we start with the local-equilibrium one $P_{\mathrm{lc}}(\Gamma)$ at $t=0$ assuming a stepwise boundary-temperature change. The relaxation $P_{\mathrm{lc}} \rightarrow P_{\mathrm{st}}$ should take place as microscopic events in a microscopic time in the preexisting temperature gradient, as was discussed by Mori ${ }^{12}$. On the other hand, the relaxation from $P_{\mathrm{e}}$ occurs slowly on the time scale of $t_{D}$. Here, for the choice $P(\Gamma, 0)=P_{\mathrm{lc}}(\Gamma)$, the dissipation function is given by $\mathcal{D}(t)$ in Eq.(112). In fact, from Eqs.(110)-(112), time derivative of Eq.(92) becomes

$$
\frac{d}{d t}\left[\beta \mathcal{H}(t)+\Psi(t)+\sum_{K} \frac{\gamma}{2} \zeta_{K}(t)^{2}\right]=\Lambda(t)+\dot{\mathcal{D}}(t),
$$

where $\Psi(t)=\Psi\left(\Gamma_{t}\right)$. This again leads to Eq.(116). Here, use is made of the approximation in the second line of Eq.(12), which is valid for large $H$.

The counterparts of Eqs.(96) and (108) are given by

$$
\begin{aligned}
& P(\Gamma, t)=P_{\mathrm{lc}}(\Gamma) \exp [\mathcal{D}(t)], \\
& P\left(\Gamma^{*}, t\right)=P_{\mathrm{lc}_{\mathrm{c}}}(\Gamma) \exp [-\mathcal{D}(t)] .
\end{aligned}
$$

As the counterpart of Eq.(109), Eq.(120) yields

$$
\bar{J}_{h}^{z}(z, t)=-\left\langle J_{h}^{z}(\boldsymbol{r}, 0) e^{-\mathcal{D}(t)}\right\rangle_{\mathrm{ls}},
$$

which tends to $-\lambda \mathcal{T}$ homogeneously in a short time in contrast to $\bar{J}_{h}^{z}(z, t)$ in Eq.(109). Thus, we find

$$
\begin{aligned}
& P_{\mathrm{st}}(\Gamma)=P_{\mathrm{lc}}(\Gamma) \exp \left[\mathcal{D}\left(t_{\mathrm{lc}}\right)\right] \\
& P_{\mathrm{st}}\left(\Gamma^{*}\right)=P_{\mathrm{lc}}(\Gamma) \exp \left[-\mathcal{D}\left(t_{\mathrm{lc}}\right)\right]
\end{aligned}
$$

where $t_{\mathrm{lc}}$ is taken to be longer than the relaxation time of $P_{\mathrm{lc}} \rightarrow P_{\mathrm{st}}$. In Eqs.(119) and (121) we use backward trajectories $\Gamma_{-s}(0<s<t)$ with $\Gamma_{0}=\Gamma$ and

$$
\mathcal{D}(t)=-\gamma_{\mathrm{a}} \int_{0}^{t} d s \mathcal{G}(-s)
$$


where we change $\mathcal{G}(s)$ in Eq.(112) to $\mathcal{G}(t-s)$ and shift the time origin by $-t$. In Eqs.(120) and (123), we use forward trajectories $\Gamma_{s}(0<s<t)$ with $\Gamma_{0}=\Gamma$.

We can also assume that $P_{\text {st }}(\Gamma)$ is given by the time average of $P(\Gamma, t)$ in a time interval with width longer than $t_{\mathrm{lc}}$. Then, the Laplace transformation $\int_{0}^{\infty} d t e^{-\epsilon t} P(\Gamma, t)$ tends to $P_{\mathrm{st}}(\Gamma) / \epsilon$ for $0<\epsilon \ll t_{\mathrm{lc}}^{-1}$. With the aid of $\epsilon e^{-\epsilon t}=-d\left(e^{-\epsilon}\right) / d t$, we obtain

$$
\begin{aligned}
& P_{\mathrm{st}}(\Gamma)=P_{\mathrm{lc}}(\Gamma)\left[1-\int_{0}^{\infty} d t e^{-\epsilon t} \mathcal{G}(-t) \gamma_{\mathrm{a}} e^{\mathcal{D}(t)}\right] \\
& P_{\mathrm{st}}\left(\Gamma^{*}\right)=P_{\mathrm{lc}}(\Gamma)\left[1+\int_{0}^{\infty} d t e^{-\epsilon t} \mathcal{G}(t) \gamma_{\mathrm{a}} e^{-\mathcal{D}(t)}\right]
\end{aligned}
$$

which readily give the linear forms (69) and (70).

In the early literature ${ }^{13}-15,17$, the steady-state distribution was expressed in the form of Eq.(122) for simple fluids, where $t_{\mathrm{lc}}$ was pushed to $\infty$. In particular, Kawasaki and Gunton ${ }^{15}$ studied sheared steady states with $P_{\mathrm{st}} / P_{\mathrm{lc}}=\exp \left[-\beta \int_{0}^{\infty} d t \int d \boldsymbol{r} \Pi_{z x}(\boldsymbol{r},-t) \dot{\gamma}\right]$, where $\Pi_{z x}$ is the shear stress and $\dot{\gamma}$ is the shear rate. They calculated the nonlinear shear viscosity due to the modecoupling effect, where the life times of the shear modes are cut off by applied shear at long wavelengths 4 .

\section{SUMMARY AND REMARKS}

We have presented a microscopic theory of applying a heat flow from thermostatted boundary walls and have derived Green's expression for the thermal conductivity $\lambda$ in the bulk. Our theory is based on the surface-tobulk connecting relationship in Eqs.(13) and (25). We give only the boundary tempeatures and do not assume a constant temperature gradient in the interior, so our method is applicable to any inhomogeneous systems. We summarize our main results as follows.

(i) In Sec.II, we have explained our system composed of unbound particles in the cell, those bound to the boundary layers, and thermostats attached to the layers. We have introduced the heat flows $\mathcal{I}_{K}$ from the bound particles to the unbound ones in Eq.(11) and those $\mathcal{J}_{K}$ from the thermostats to the particles in Eq.(23). They have bulk expressions in Eqs.(13) and (25) as key relations.

(ii) In Sec.III, we have derived linear response relations to small boundary temperature changes $\delta T_{K}(t)$ from the Liouville equation. Their surface expressions have been given in Eqs.(41) and (42) in terms of the time-correlation functions $\left\langle\mathcal{B}(t) \mathcal{J}_{K}(0)\right\rangle_{\mathrm{e}}$. Using Eq. (25), we have also obtained the bulk response expressions composed of localequilibrium and dissipative parts. We have also calculated the nonequilibrium average of the Evans-Searles dissipation function $\Omega(t)^{27,34,36,38}$ generally in terms of the hydrodynamic variables in Eqs.(56) and (61) and explicitly for a stepwise temperature change in Eq.(62).

(iii) In Sec.IV, we have examined the phase-space distribution $P(\Gamma, t)$ in the nonlinear regime. First, we have summarized salient results when the initial distribution $P(\Gamma, 0)$ is the equilibrium one. In particular, for $\delta T_{\text {top }}(t)=\delta T_{\text {bot }}(t)$, we have obtained simple results including Eqs.(101) and (102). Furthermore, using Eq.(25), we have introduced the local-equilibrium distribution $P_{\mathrm{lc}_{\mathrm{c}}}(\Gamma)$ in Eq.(114). In the case $P(\Gamma, 0)=P_{\mathrm{lc}_{\mathrm{c}}}(\Gamma)$, we have obtained the steady-state one $P_{\text {st }}(\Gamma)$ in the McLennan-Zubarev form ${ }^{13,14}$ in Eqs.(122) and (123).

(iv) We have examined the linear relaxations of the hydrodynamic variables in Appendix A and the dissipattion function in in Appendix B after a boundary temperature change. These results enable us to calculate the time-correlation functions $\left\langle\mathcal{B}(t) \mathcal{J}_{K}(0)\right\rangle_{\mathrm{e}}$. We have presented a theory of the long-range correlation in the canonical ensemble in Appendix C, which leads to the local-equilibrium response in steady states. In Appendix $\mathrm{D}$, we have calculated $\Omega(t)$ including $T$-dependence of $\tau_{\mathrm{h}}$.

We make some remarks as follows. (1) Though we have used Nosé-Hoover thermostats ${ }^{42,43}$, our results should be independent of the thermostating method on longtime scales $\left(\gg \tau_{\mathrm{h}}\right)$. (2) We should examine the thermal boundary resistance at a solid-fluid interface in more detail. Its Green-Kubo type formula ${ }^{21,50}$ has not yet been firmly established from our viewpoint. (3) It is of great interest to generalize our results to multi-component fluids, where the thermo-diffusion effect is crucial. (4) Our scheme is applicable to systems in the presence of twophase interfaces ${ }^{22,50}$ and to mesoscopically heterogeneous systems such as glasses and polycrystals. (5) In our previous paper on shear strains ${ }^{18}$ we examined only the linear response. We should further examine sheared states in the presence of thermostats. (6) Numerical study of the relaxation $P_{\mathrm{lc}} \rightarrow P_{\mathrm{st}}$ should be informative, which is easy particularly for one-dimensional systems.

\section{Acknowledgments}

I would like to thank Takeshi Kawasaki for valuable discussions on the strain effect in glasses leading to this work. I am also indebted to Hisao Hayakawa for useful correspondence on the fluctuation theorems.

\section{Appendix A: Thermal relaxation after a bound- ary temperature change at a fixed volume}

To examine slow dynamics in the film region $0<$ $z<H$, we treat averaged smooth quantities obeying the linearlized hydrodynamic equations on timescales much longer than the typical molecular time $t_{\mathrm{m}}$. We start with the heat conduction equation ${ }^{6,7}$,

$$
n T \frac{\partial}{\partial t} \delta s=\lambda \nabla_{z}^{2} \delta T,
$$

without significant Kapitza resistance. Here, $\delta s$ is the deviation of the entropy per particle related to that of the temperature $\delta T$ and that of the pressure $\delta p$ by

$$
n T \delta s=C_{p}\left[\delta T-(\partial T / \partial p)_{s} \delta p\right] .
$$

Here, $n T \delta s$ is the average deviation of $\hat{q}$ in Eq.(4) and $\delta p$ is that of $\hat{p}$ in Eq.(8) slightly away from equilibrium. 
They are related to $\chi_{a K}(z, t)=\left\langle\hat{a}(\boldsymbol{r}, t) \mathcal{J}_{K}(0)\right\rangle_{\mathrm{e}}$ for $\hat{a}=\hat{q}$ and $\hat{p}$ (see Sec.IIIA). In the linear order, the temperature deviation $\delta T$ is defined by Eq.(A2). The average boundary heat fluxes $\mathcal{I}_{K}(t)\left(\cong \mathcal{J}_{K}(t)\right)$ are written as

$$
\mathcal{I}_{\text {top }}(t)=A \lambda \delta T^{\prime}(H, t), \mathcal{I}_{\text {bot }}(t)=-A \lambda \delta T^{\prime}(0, t),
$$

where $A$ is the surface area and $\delta T^{\prime}(z, t)=\partial \delta T / \partial z$.

Let the boundary temperatures be changed by constant $\delta T_{K}$ in a time range $\left[0, t_{\mathrm{ex}}\right]$ with $t_{\mathrm{ex}}<t_{\mathrm{a}}=$ $H / c$. For example, we can assume the linear increase: $\delta T_{K}(t) / \delta T_{K}=t / t_{\mathrm{ex}}$ for $0<t<t_{\mathrm{ex}}$.

Initial stage. For $t_{\mathrm{m}} \ll t<t_{\mathrm{a}}$, we treat the thermal diffusion near the walls. Along the $z$ axis, $\delta T(z, t)$ decreases from $\delta T_{K}(t)$ at the boundaries and decays to 0 far from them on the diffusion length $\sqrt{D t}$. Thus, for $0<t<t_{\mathrm{a}}, \mathcal{I}_{K}(t)$ and $\delta \mathcal{H}(t)$ increase as

$$
\begin{aligned}
& \mathcal{I}_{K}(t) \sim A \lambda \delta T_{K}(t) / \sqrt{D t} \\
& \delta \mathcal{H}(t) \sim \sum_{K} A \lambda \delta T_{K}(t) \sqrt{t / D} .
\end{aligned}
$$

See Eq.(63) for the corresponding response functions.

Intermediate and final stages. For $t \gg t_{\mathrm{a}}, \delta p$ is known to be homogenized after repeated sound traversals in the cell (the piston effect) $\underline{4}+\underline{4}-46$, where $\delta p$ is equal to the space average of $(\partial p / \partial T)_{n} \delta T+(\partial p / \partial n)_{T} \delta n$. Thus,

$$
\delta p(t)=(\partial p / \partial T)_{n}\langle\delta T\rangle_{\mathrm{sp}}(t)
$$

where $\langle\delta T\rangle_{\mathrm{sp}}=\int_{0}^{H} d z \delta T(z, t) / H$. As a result, the temperature increases by $(\partial T / \partial p)_{s} \delta p$ adiabatically throughout the cell. This effect is amplified near the criticality, where the ratio $(\partial T / \partial p)_{s} /(\partial T / \partial p)_{n}=1-\gamma_{\mathrm{s}}^{-1}$ is close to 1 with $\gamma_{\mathrm{s}}=C_{p} / C_{V} \gg 1$. The inhomogeneous part of $\delta T$ near the walls is governed by the thermal diffusion.

For $t \gg t_{\mathrm{a}}$, we can use Eq.(A6) to calculate the Laplace transformation (LT) of $\delta T(z, t)$ defined by

$$
F_{T}(z, \omega)=\int_{0}^{\infty} d t e^{-\omega t} \delta T(z, t)
$$

which is valid for $\omega \ll t_{\mathrm{a}}^{-1}$. Some calculations give 44

$$
F_{T}=\left[1+\frac{\cosh v-\cosh u}{\varphi(u)}\right] \frac{\delta T_{\mathrm{m}}}{\omega}+\frac{H \sinh v}{2 \sinh u} \cdot \frac{\mathcal{T}}{\omega},
$$

where $\delta T_{\mathrm{m}}$ and $\mathcal{T}$ are given in Eqs.(35) and (36) and

$$
\begin{gathered}
u=H(\omega / 4 D)^{1 / 2}=\left(t_{D} \omega\right)^{1 / 2}, \\
v=u(2 z / H-1), \\
\varphi(u)=\cosh u+\left(\gamma_{\mathrm{s}}-1\right) u^{-1} \sinh u .
\end{gathered}
$$

Here, $F_{T}(z, \omega)$ depends on $z$ through $v$ with $v= \pm u$ at $z=0$ and $H$. From Eq.(A8) its space average is given by

$$
\left\langle F_{T}\right\rangle_{\mathrm{sp}}=\left(\frac{\partial T}{\partial p}\right)_{n} F_{p}=\frac{\gamma_{\mathrm{s}} \sinh u}{u \varphi(u)} \cdot \frac{\delta T_{\mathrm{m}}}{\omega}
$$

where $F_{p}(\omega)$ is the LT of $\delta p(t)$ in Eq.(A6). The LTs: $F_{K}(\omega)=\int_{0}^{\infty} d t e^{-\omega t} \mathcal{I}_{K}(t)$ are also given by

$$
F_{K}(\omega)=A \lambda t_{D}\left[\frac{2 \sinh u}{H u \varphi(u)} \delta T_{\mathrm{m}} \pm \frac{\mathcal{T}}{u \tanh u}\right]
$$

where + is for $K=$ top and - is for $K=$ bot. For $\gamma_{\mathrm{s}} \gg 1$, we have near-critical behavior $\varphi \cong\left(\gamma_{\mathrm{s}}-1\right) / u \gg 1$ for $1 \ll u \ll \gamma_{\mathrm{s}}$ (see Eqs.(B7) and (B8)).

We remark the following. (i) We find $F_{T}(z, \omega) \rightarrow$ $\delta \bar{T}(z) / \omega$ as $\omega \rightarrow 0$ and $\delta T(z, t) \rightarrow \delta \bar{T}(z)$ as $t \rightarrow \infty$, where $\delta \bar{T}(z)$ is given in Eq.(54) in the steady state. (ii) For $u \gg 1$, we have $\omega\left\langle F_{T}\right\rangle_{\mathrm{sp}} \cong \delta T_{\mathrm{m}} \gamma_{\mathrm{s}} /\left(u+\gamma_{\mathrm{s}}-1\right)$. Thus, for $\gamma_{\mathrm{s}} \gg 1$, we have $\langle\delta T\rangle_{\mathrm{sp}}(t) \cong \delta T_{\mathrm{m}}$ for $t \gtrsim t_{1}$, where

$$
t_{1}=\left(\gamma_{\mathrm{s}}-1\right)^{-2} H^{2} / 4 D \ll t_{D} .
$$

in particular, for $\mathcal{T}=0$, Thus, thermalization occurs at $t \sim t_{1}$. We assume $t_{1} \gg t_{\mathrm{a}}$, which holds for $H \gg \gamma_{\mathrm{s}}^{2} D / c$, while Eq.(A8) holds even in the reverse case $t_{1} \lesssim t_{\mathrm{a}}$. (iii) The second term in Eq.(A8) gives rise to the temperature relaxation approaching the linear profile $\mathcal{T}(z-H / 2)$ diffusively on the timescale of $t_{D}$.

\section{Appendix B: Dissipation function in hydrody- namic regime}

In this appendix, we first derive Eq.(56). From Eqs.(A1)-(A3) the hydrodynamic average of the dissipation function in Eq.(50) is written as

$$
\begin{aligned}
& \frac{\bar{\Omega}(t)}{A}=\frac{\lambda}{k_{B} T^{2}} \int_{0}^{t} d t\left[\left(\delta T \delta T^{\prime}\right)_{z=H}-\left(\delta T \delta T^{\prime}\right)_{z=0}\right] \\
& =\frac{1}{k_{B} T} \int_{0}^{t} d t \int_{0}^{H} d z\left[n \frac{\partial \delta s}{\partial t} \delta T+\frac{\lambda}{T}\left(\delta T^{\prime}\right)^{2}\right] .
\end{aligned}
$$

To derive the second line, we change the integrand in the first line to $\int_{0}^{H} d z\left[\delta T \delta T^{\prime \prime}+\left(\delta T^{\prime}\right)^{2}\right]$ and use Eq.(A1). We also calculate the time derivative of $(\Delta S)_{2}$ in Eq.(57) as

$$
\frac{d}{d t} \int d \boldsymbol{r}(\Delta S)_{2}=-\int d \boldsymbol{r}\left[\frac{n}{T} \frac{\partial \delta s}{\partial t} \delta T+\frac{\partial \delta n}{\partial t} \frac{\delta p}{n T}\right],
$$

where $C_{V} \delta T=n T \delta s+T(\partial p / \partial T)_{n} \delta n / n$. Here, the space integral of $(\partial \delta n / \partial t) \delta p / n$ is changed to that of $\boldsymbol{v} \cdot \nabla \delta p=$ $\boldsymbol{v} \cdot\left[-\rho \dot{\boldsymbol{v}}+\nabla \cdot \overleftrightarrow{\sigma}_{\mathrm{v}}\right]$ from $\partial(\delta n) / \partial t=-n \nabla \cdot \boldsymbol{v}$, where $\stackrel{\leftrightarrow}{\sigma}_{\mathrm{v}}$ is the viscous stress tensor. Thus, we are led to Eq.(56).

Second, we explicitly calculate $\bar{\Omega}(t)$. In the initial stage $0<t<t_{\mathrm{a}}$, the bottom and top disturbed regions are separated, so Eq.(A4) yields

$$
\bar{\Omega}(t) / A \sim \lambda \sqrt{t / D} \sum_{K}\left[\delta T_{K}(t)\right]^{2} / k_{B} T^{2}
$$

For $t \gg t_{\mathrm{a}}, \mathrm{Eq},(\mathrm{A} 8)$ gives the LT of $\bar{\Omega}(t)$ in the form,

$$
F_{\Omega}(\omega)=\frac{V \lambda t_{D}}{k_{B} T^{2} \omega}\left[\frac{4 \sinh u}{u \varphi(u)} \cdot \frac{\left(\delta T_{\mathrm{m}}\right)^{2}}{H^{2}}+\frac{\mathcal{T}^{2}}{u \tanh u}\right]
$$


whose inverse LT yields Eq.(62). Using $\tau=t / t_{D}$ we have

$$
\begin{gathered}
\psi_{1}(\tau)=\sum_{\ell \geq 1} \frac{2 \gamma_{\mathrm{s}}}{a_{\ell}^{2}+\gamma_{\mathrm{s}}\left(\gamma_{\mathrm{s}}-1\right)}\left[1-\exp \left(-a_{\ell}^{2} \tau\right)\right], \\
\psi_{2}(\tau)=\sum_{\ell \geq 1} \frac{2}{\pi^{2} \ell^{2}}\left[1-\exp \left(-\pi^{2} \ell^{2} \tau\right)\right] .
\end{gathered}
$$

In Eq.(B5), $a_{\ell}(\ell \geq 1)$ are the solutions of $\tan a_{\ell}=$ $-a_{\ell} /\left(\gamma_{\mathrm{s}}-1\right)$ with $\ell-1 / 2<a_{\ell} / \pi<\ell$. The inverse LTs of $t_{D} \gamma_{\mathrm{s}} \sinh u / u \varphi(u)$ and $t_{D} /(u \tanh u)-1 / \omega$ are $\psi_{1}^{\prime}=d \psi_{1} / d \tau$ and $\psi_{2}^{\prime}=d \psi_{2} / d \tau$, respectively, which indicate $\psi_{1}(\infty)=1$ and $\psi_{2}(\infty)=1 / 3$. For $\gamma_{\mathrm{s}} \gg 1, \psi_{1}$ depends on $\gamma_{\mathrm{s}}$ in a singular manner as 44

$$
\begin{aligned}
\psi_{1}(\tau) & \cong 2\left(\gamma_{\mathrm{s}}-1\right)(\tau / \pi)^{1 / 2} \quad\left(t<t_{1}\right) \\
& \cong 1-\left(\gamma_{\mathrm{s}}-1\right)^{-1} /(\pi \tau)^{1 / 2} \quad\left(t_{1}<t<t_{D}\right) .
\end{aligned}
$$

Thus, $\psi_{1}$ appraoches to 1 for $t \sim t_{1}$ (the piston effect).

\section{Appendix C: Long-range correlation in finite systems in canonical ensemble}

We examine the long-range correlations in a finite system in the $T-N-V$ canonical ensemble $47,56-58$, which are absent in the $T-\mu-V$ grand canonical ensemble. We treat macroscopic one-component fluids, but extension to multi-component fluids ${ }^{56-58}$ is straightforward. The average $\langle\cdots\rangle_{\mathrm{e}}$ is taken at fixed $V, T$, and $N=n V$.

In the canonical ensemble, the density correlation function $g\left(\boldsymbol{r}, \boldsymbol{r}^{\prime}\right)=\left\langle\delta \hat{n}(\boldsymbol{r}) \delta \hat{n}\left(\boldsymbol{r}^{\prime}\right)\right\rangle_{\mathrm{e}}$ in the bulk is of the form,

$$
g\left(\boldsymbol{r}, \boldsymbol{r}^{\prime}\right)=n \delta\left(\boldsymbol{r}-\boldsymbol{r}^{\prime}\right)+n^{2} g\left(\left|\boldsymbol{r}-\boldsymbol{r}^{\prime}\right|\right)-k_{B} T n^{2} \kappa_{T} / V,
$$

where $g(r)$ is the pair correlation function and $\kappa_{T}$ is the isothermal compressibility. Space integration of Eq.(C1) in the cell vanishes from $1+n \int d \boldsymbol{r} g(r)=k_{B} T n \kappa_{T}$.

For any density variables $\hat{a}(\boldsymbol{r})$ and $\hat{b}(\boldsymbol{r})$, we consider their deviations $\delta \hat{a}(\boldsymbol{r})=\hat{a}(\boldsymbol{r})-a$ and $\delta \hat{b}(\boldsymbol{r})=\hat{b}(\boldsymbol{r})-b$, where the averages $a=\langle\hat{a}\rangle_{\mathrm{e}}$ and $b=\langle\hat{b}\rangle_{\mathrm{e}}$ are thermodynamic quantities. In the canonical ensemble, we have

$$
\left\langle\delta \hat{a}(\boldsymbol{r}) \delta \hat{b}\left(\boldsymbol{r}^{\prime}\right)\right\rangle_{\mathrm{e}}=g_{a b}\left(\boldsymbol{r}-\boldsymbol{r}^{\prime}\right)-k_{B} T n^{2} \kappa_{T} D_{a b} / V,
$$

where $g_{a b}\left(\boldsymbol{r}-\boldsymbol{r}^{\prime}\right)$ is a short-ranged function and

$$
D_{a b}=\left(\frac{\partial a}{\partial n}\right)_{T}\left(\frac{\partial b}{\partial n}\right)_{T}=\frac{1}{n \kappa_{T}}\left(\frac{\partial a}{\partial p}\right)_{T}\left(\frac{\partial b}{\partial n}\right)_{T} .
$$

Here, if $\hat{b}=\hat{q}$, Eq.(79) follows from $n^{2}(\partial s / \partial n)_{T}=$ $-(\partial p / \partial T)_{n}$. If $\hat{b}=\hat{p}$, the second term of Eq.(C2) is $-n k_{B} T(\partial a / \partial n)_{T} / V$. If $\hat{b}$ is the temperature fluctuation $\delta \hat{T} \equiv C_{V}^{-1} \delta \hat{q}+(\partial T / \partial n)_{s} \delta \hat{n}$, we have $D_{a b}=0$.

A general theory of Lebowitz et al. ${ }^{47}$ can yield Eq.(C2), but we here present its simple derivation. We divide the cell into regions $A$ and $B$ with volumes $V_{A}$ and $V_{B}=$ $V-V_{A}$, where $\boldsymbol{r}$ is in $\mathrm{A}$ and $\boldsymbol{r}^{\prime}$ is in B far from the boundaries. We examine the fluctuation of the particle number $N_{A}$ in A around $\bar{N}_{A}=n V_{A}$. Then, for each $N_{A}$, the conditional average of $\hat{a}(\boldsymbol{r})$ is $a\left(T, N_{A} / V_{A}\right)$, so

$$
\delta \hat{a}(\boldsymbol{r})=a\left(T, N_{A} / V_{A}\right)-a(T, n) \cong\left(\frac{\partial a}{\partial n}\right)_{T} \frac{\delta N_{A}}{V_{A}}
$$

where $\left|\delta N_{A}\right| \ll \bar{N}_{A}$. In B, we have $N_{B}=\bar{N}_{B}-\delta N_{A}$ with $\bar{N}_{B}=n V_{B}$ so that $\delta \hat{b}\left(\boldsymbol{r}^{\prime}\right)=-(\partial b / \partial n)_{T} \delta N_{A} / V_{B}$. Thus,

$$
\left\langle\delta \hat{a}(\boldsymbol{r}) \delta \hat{b}\left(\boldsymbol{r}^{\prime}\right)\right\rangle_{\mathrm{e}}=-D_{a b} \sum_{\delta N_{A}} P\left(N_{A}\right)\left(\delta N_{A}\right)^{2} / V_{A} V_{B}
$$

where $P\left(N_{A}\right)$ is the distribution of $N_{A}$ proportional to $\exp \left[-F\left(T, N_{A}\right) / k_{B} T-F\left(T, N-N_{A}\right) / k_{B} T\right]$, where $F$ is the Helmholtz free energy. Up to second order, we find

$$
P\left(N_{A}\right) \propto \exp \left[-\left(V / 2 k_{B} T \kappa_{T} \bar{N}_{A} \bar{N}_{B}\right)\left(\delta N_{A}\right)^{2}\right]
$$

Using this Gaussian distribution we sum over $\delta N_{A}$ in Eq.(C5) to obtain the second term in Eq.(C2).

Finally, we write the space integral of the first term in Eq.(C2) as $\langle\hat{a}: \hat{b}\rangle \equiv \int d \boldsymbol{r} g_{a b}(\boldsymbol{r})$ in the grand canonical ensemble. Then, we have $\langle\hat{a}: \hat{n}\rangle=n k_{B} T(\partial a / \partial p)_{T}$ and $\langle\hat{a}: \hat{q}\rangle=k_{B} T^{2}(\partial a / \partial T)_{p}$ ․ We can now rewrite the second term in Eq.(C2) as $-\langle\hat{a}: \hat{n}\rangle\langle\hat{b}: \hat{n}\rangle / V\langle\hat{n}: \hat{n}\rangle$..

\section{Appendix D: Dissipation function including temperature-dependence of $\tau_{\mathrm{h}}$}

We here derive the dissipation function $\Omega(t)$ for $T$ dependent $\tau_{\mathrm{h}}$ in the nonlinear theory, where $\tau_{\mathrm{h}}=$ $\tau_{\mathrm{h}}\left(T_{K}(t)\right)$ depends on $t$ and $K$. From Eq.(17), we have

$$
\dot{\zeta}_{K}(t)=\left[2 E_{K}(t)-d M k_{B} T_{K}(t)\right] / Q_{K}(t),
$$

where $Q_{K}(t)=\left[\tau_{\mathrm{h}}\left(\left(T_{K}(t)\right)\right]^{2} d M k_{B} T_{K}(t)\right.$. Evans and Searles $27,36,38$ treated $Q_{K}(t)$ as a constant $Q$.

For $P(\Gamma, 0)=P_{\mathrm{e}}(\Gamma)$, the first line of Eq.(93) gives

$$
\begin{aligned}
& \dot{\Omega}(t)=2 \sum_{K} \delta \beta_{K}(t) E_{K}(t) \zeta_{K}(t) \\
& -\sum_{K}\left[\beta_{K}(t) Q_{K}(t)-\beta Q_{K}(0)\right] \zeta_{K}(t) \dot{\zeta}_{K}(t) .
\end{aligned}
$$

The first term yields Eq.(50) from Eq.(23), while the second term arises from $T$-dependence of $\tau_{\mathrm{h}}$ from $\beta_{K}(t) Q_{K}(t)=d M\left[\tau_{\mathrm{h}}\left(T_{K}(t)\right)\right]^{2}$. In particular, if $Q_{K}(t)=$ $Q_{K}(0)$ or if $\tau_{\mathrm{h}}^{2} \propto T^{-1}$, Eqs.(D1) snd (D2) yield

$$
\dot{\Omega}(t)=-d M \sum_{K} \zeta_{K}(t) \delta T_{K}(t) / T
$$

whose time-integration gives the Evans-Searles $\Omega(t)$ for heat conduction 27,36,38. However, for any $T$-dependence of $\tau_{\mathrm{h}}$, the difference between our $\Omega(t)$ and theirs is negligible for $t \gg \tau_{\mathrm{h}}$, since the second term in Eq.(D2) is proportional to $d \zeta_{K}(t)^{2} / d t$. In the linear regime, Eq.(D2) gives Eq. (48) from $\tau_{\mathrm{h}}\left(T_{K}\right)-\tau_{\mathrm{h}}(T) \cong\left(\partial \tau_{\mathrm{h}} / \partial T\right) \delta T_{K}$. In particular, we have $\mathcal{J}_{K}^{\prime}(t)=-d M k_{B} T \zeta_{K}(t)$ for $\tau_{\mathrm{h}}^{2} \propto T^{-1}$. 
1 R. Zwanzig, "Time-correlation functions and transport coefficients in statistical mechanics," Annu. Rev. Phys. Chem. 16, 67-101 (1965).

2 J.-P. Hansen and I. R. Mcdonald, Theory of Simple Liquids (Academic, 2006).

3 D. J. Evans and G. P. Morriss, Statistical Mechanics of Nonequilibrium Liquids (Academic, London, 1990).

4 A. Onuki, Phase Transition Dynamics (Cambridge University Press, Cambridge, 2002).

5 M.S. Green, "Markoff random processes and the statistical mechanics of time-dpendent phenomena. II. Irreversible processes in fluids," J. Chem. Phys. 22, 398-413 (1954).

6 L.P. Kadanoff and P.C. Martin, "Hydrodynamic Equations and Correlation Functions," Ann. of Phys. 24, 419-469 (1963)

7 L.D. Landau and E.M. Lifshitz, Fluid Mechanics (Pergamon, New York, 1959).

${ }^{8}$ L. Onsager, "Reciprocal relations in irreversible processes.I.," Phys. Rev. 37, 405-426 (1931).

9 R. Zwanzig, "Memory effects in irreversible thermodynamics," Phys. Rev. 124, 983-992 (1961).

10 H. Mori, "Transport, Collective Motion, and Brownian Motion," Progr. Theoret. Phys. 33, 423-455 (1965)

11 R. Kubo, "The fluctuation-dissipation theorem," Rep. Prog. Phys. 29, 255-284 (1966).

12 H. Mori, "Statistical-Mechanical Theory of Transport in Fluids," Phys. Rev. 112, 1829-1842 (1958).

13 J. A. McLennan, "Nonlinear Effects in Transport Theory," Phys. Fluids 4, 1319-1324 (1961).

14 D. N. Zubarev, Dokl. Akad. Nauk. SSSR 140, 92-95 (1961) ["The Statistical Operator for Nonequilibrium Systems,"Sov. Phys.-Doklady 6, 776 (1962)]; "The Method of the Non-Equilibrium Statistical Operator and its Applications. I," Fortschritte der Physik 18, 125-147 (1970).

15 K. Kawasaki and J. D. Gunton, "Theory of Nonlinear Transport Processes: Nonlinear Shear Viscosity and Normal Stress Effect," Phys. Rev., A 8, 2048-2064 (1973).

16 R. Kubo, "Statistical-mechanical theory of irreversible processes.I. General theory and simple applications to magnetic and conduction problems," J. Phys. Soc. Jpn. 12, 570-586 (1957).

17 I. Procaccia, D. Ronis, M. A. Collins, J. Ross, and I. Oppenheim, "Statistical mechanics of stationary states. I. Formal theory," Phys. Rev. A 19, 1290-1306 (1979).

18 A. Onuki and T. Kawasaki, "Theory of applying shear strains from boundary walls : linear response in glasses," J. Chem. Phys. 150, 124504 (2019).

19 J. Petravic and P. Harrowell, "Linear response theory for thermal conductivity and viscosity in terms of boundary fluctuations," Phys. Rev. E 71, 061201 (2005); "Equilibrium calculations of viscosity and thermal conductivity across a solid-liquid interface using boundary fluctuations," J. Chem. Phys. 128, 194710 (2008).

${ }^{20}$ F. Bonetto, J.L. Lebowitz, L. Rey-Bellet, "Fourierfs law: a challenge to theorists", Mathematical Physics 2000, 128150, (Imperial College Press, London, 2000).

21 J.-L. Barrat and F. Chiaruttini, "Kapitza resistance at the liquid-solid interface," Mol. Phys. 101, 1605-1610 (2003).

22 A. Røsjorde, D. W. Fossmo, D. Bedeaux, S. Kjelstrup, and B. Hafskjold, "Non-equilibrium molecular dynamics calculation of heat conduction in liquid and through liquid- gas interface," J. Colloid and Interface Sci. 232, 178-185 (2000).

23 T. Hamanaka, R. Yamamoto, and A. Onuki, "Molecular dynamics simulation of heat conduction in near-critical fluids," Phys. Rev. E 71, 011507 (2005).

24 S. Lepri, R. Livi, and A. Politi, "Energy transport in anharmonic lattices close to and far from equilibrium," Physica D 119, 140-147 (1998); "Thermal conduction in classical low-dimensional lattices," Phys. Rep. 377, 1-80 (2003).

25 M. M. Sano and K. Kitahara, "Thermal conduction in a chain of colliding harmonic oscillators revisited," Phys. Rev. E 64, 056111 (2001); K. Aoki and D. Kusnezov, "Fermi-Pasta-Ulam b Model: Boundary Jumps, Fourier's Law, and Scaling," Phys. Rev. Lett. 86, 4029 (2001).

26 A. Dhar, "Heat transport in low-dimensional systems," Adv. in Phys. 57, 457-537 (2008).

27 D. J. Evans and D. J. Searles, "Equilibrium microstates which generate second law violating steady states," Phys. Rev. E 50, 1645 (1994); "The Fluctuation Theorem," Adv. in Phys. 51, 1529-15851 (2002).

28 G. Gallavotti and E. G. D. Cohen, "Dynamical Ensembles in Stationary States," J. Stat. Phys. 80, 931-970 (1995); "Dynamical Ensembles in Nonequilibrium Statistical Mechanics,"Phys. Rev. Lett. 74, 2694 (1995).

29 C. Jarzynski, "Nonequilibrium equality for free energy differences," Phys.Rev. Lett. 78, 2690-93 (1997).

30 C. Jarzynski, "Hamiltonian Derivation of a Detailed Fluctuation Theorem," J. Stat. Phys. 98, 77-102 (2000).

31 G. E. Crooks, "Entropy production fluctuation theorem and the nonequilibrium work relation for free energy differences," Phys. Rev. E 60, 2721 (1999); "Path-ensemble averages in systems driven far from equilibrium," ibid. $\mathbf{6 3}$, 2361-2366 (2000).

32 J. L. Lebowitz and H. Spohn, "A Gallavotti-Cohen-Type Symmetry in the Large Deviation Functional for Stochastic Dynamics," J. Stat. Phys. 95, 333-365 (1999).

33 C. Bustamante, J. Liphardt, and F. Ritort," The nonequilibrium thermodynamics of small systems," Physics Today 58, 7, 43-48 (2005).

34 D. J. Evans, D. J. Searles, and S. R. Williams, "On the fluctuation theorem for the dissipation function and its connection with response theory," J. Chem. Phys. 128, 014504 (2008).

35 U. Seifert, "Stochastic thermodynamics, fluctuation theorems and molecular machines," Rep. Prog. Phys. 75, 126001 (2012).

36 D. J. Searles and D. J. Evans, "Fluctuation Theorem for Heat Flow," Int. J. Thermophys. 22, 123 (2001).

37 C. Jarzynski D. K.Wójcik, "Classical and Quantum Fluctuation Theorems for Heat Exchange," Phys. Rev. Lett. 92, 230602 (2004).

38 S. R. Williams, D. J. Searles, and D. J. Evans, "Nonequilibrium Free-Energy Relations for Thermal Changes," Phys. Rev. Lett. 100, 250601 (2008).

39 T. S. Komatsu, N. Nakagawa, S. Sasa, and H. Tasaki, "Entropy and Nonlinear Nonequilibrium Thermodynamic Relation for Heat Conducting Steady States", J. Stat. Phys. 134, 401-423 (2009); "Exact Equalities and Thermodynamic Relations for Nonequilibrium Steady States," ibid. 159, 1237-1285 (2015).

40 D. J. Evans, D. J. Searles, and S. R. Williams, " On the 
probability of violations of Fourier's law for heat flow in small systems observed for short times," J. Chem. Phys., 132, 024501 (2010)

${ }^{41}$ K. Kanazawa, T. Sagawa, and H. Hayakawa, "Heat conduction induced by non-Gaussian athermal fluctuations," Phys. Rev. E 87, 052124 (2013).

42 S. Nosé, "A molecular dynamics method for simulations in the canonical ensemble," Mol. Phys. 52, 255-268 (1984); "Constant temperature molecular dynamics methods," Progr. Theoret. Phys. (Kyoto), 103, 1-46 (1991).

43 W. G. Hoover, "Canonical dynamics: Equilibrium phasespace distributions," Phys. Rev. A 31, 1695-1697 (1985).

44 A. Onuki and R. A. Ferrell, "Adiabatic Heating Effect near the Gas-Liquid Critical Point," Physica A 164, 245-264 (1990);

45 Y. Garrabos, M. Bonetti, D. Beysens, F. Perrot, T. Fröhlich, P. Carlèes, and B. Zappoli, "Rlaxation of a supercritical fluid after a heat pulse in absence of gravity effects," Phys. Rev. E 57, 5665 (1998).

46 Y. Miura, S. Yoshihara, M. Ohnishi, K. Honda, M. Matsumoto, J. Kawai, M. Ishikawa, H. Kobayashi, and A. Onuki, "High-speed observation of the piston effect near the gas-liquid critical point," Phys.Rev. E 74, 010101(R) (2006).

47 J. L. Lebowitz and J. K. Percus, "Long-Range Correlations in a Closed System with Application to Nonuniform Fluids," Phys. Rev. 124, 1673-1691 (1961); J. L. Lebowitz, J. K. Percus, and L.Verlet, "Ensemble dependence of fluctuations with application to machine computations," ibid. 153, 250-254 (1967).

${ }^{48}$ H. Shiba and A. Onuki, " Plastic deformations in crystal, polycrystal, and glass in binary mixtures under shear: collective yielding, ” Phys. Rev. E 81, 051501 (2010).
49 J. H. Irving and J. G. Kirkwood, "The statistical mechanical theory of transport processes. IV. The equations of hydrodynamics." J. Chem. Phys. 18, 817-829 (1949).

50 L. Puech, G. Bonfait, and B. Castaing, "Mobility of the 3He Solid-Liquid Interface: Experiment and Theory," J. Low Temp. Phys. 62, 315-327 (1986).

51 E. Helfand, "Transport coefficients from dissipation in a canonical ensemble," Phys. Rev. 119, 1-9 (1960).

52 S. Viscardy, J. Servantie, and P. Gaspard, "Transport and Helfand moments in the Lennard-Jones fluid. II. Thermal conductivity," J. Chem. Phys. 126, 184513 (2007).

53 W. Q. Hoover, A. J. C. Ladd, and B. Moran, "High-StrainRate Plastic Flow Studied via Nonequilibrium Molecular Dynamics," Phys. Rev. Lett. 48, 1818 (1982).

${ }^{54}$ H. B. Callen, Thermodynamics (Wiley, New York,1960).

55 L.D. Landau and E.M. Lifshitz, Statistical Physics (Pergamon, New York, 1964).

56 S. K. Schnell, X. Liu, J.-M. Simon, A. Bardow, D. Bedeaux, T. J. H. Vlugt, and S. Kjelstrup, "Calculating thermodynamic properties from fluctuations at small scales," J. Phys. Chem. B 115, 10911-10918 (2011).

57 R. Cortes-Huerto, K. Kremer, and R. Potestio, "Communication: Kirkwood- Buff integrals in the thermodynamic limit from small sized molecular dynamics simulations," J. Chem. Phys. 145, 141103 (2016).

58 B. M. Rogers, "Extension of Kirkwood-Buff theory to the canonical ensemble, J. Chem. Phys. 148, 054102 (2018).

59 A. Onuki, "On fluctuations in $\mu$ space," J. Stat. Phys. 18, 475-499 (1978). See also Eq.(5.4.28) in Ref.[4].

60 J.R. Dorfman, T. R. Kirkpatrick, and J. V. Sengers, "Generic long-range correlations in molecular fluids," Ann. Rev. Phys. Chem. 45, 213-239 (1994). 